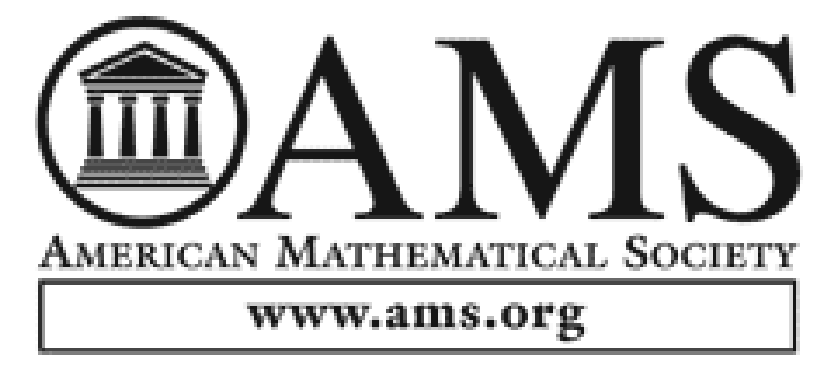

\title{
A Classification of Baire Class 1 Functions
}

Author(s): A. S. Kechris and A. Louveau

Source: Transactions of the American Mathematical Society, Vol. 318, No. 1 (Mar., 1990), pp. 209-236

Published by: American Mathematical Society

Stable URL: http://www.jstor.org/stable/2001236

Accessed: 20/05/2013 12:26

Your use of the JSTOR archive indicates your acceptance of the Terms \& Conditions of Use, available at http://www.jstor.org/page/info/about/policies/terms.jsp

JSTOR is a not-for-profit service that helps scholars, researchers, and students discover, use, and build upon a wide range of content in a trusted digital archive. We use information technology and tools to increase productivity and facilitate new forms of scholarship. For more information about JSTOR, please contact support@jstor.org. 


\title{
A CLASSIFICATION OF BAIRE CLASS 1 FUNCTIONS
}

\author{
A. S. KECHRIS AND A. LOUVEAU
}

\begin{abstract}
We study in this paper various ordinal ranks of (bounded) Baire class 1 functions and we show their essential equivalence. This leads to a natural classification of the class of bounded Baire class 1 functions $\mathscr{B}_{1}$ in a transfinite hierarchy $\mathscr{B}_{1}^{\xi} \quad\left(\xi<\omega_{1}\right)$ of "small" Baire classes, for which (for example) an analysis similar to the Hausdorff-Kuratowski analysis of $\Delta_{2}^{0}$ sets via transfinite differences of closed sets can be carried out. The notions of pseudouniform convergence of a sequence of functions and optimal convergence of a sequence of continuous functions to a Baire class 1 function $f$ are introduced and used in this study.
\end{abstract}

Let $E$ be a compact metrizable space. Our aim is to analyze, from a descriptive theoretic point of view, the set of Baire class 1 functions on $E$. Various ordinal assignments have been proposed for Baire class 1 functions, especially in Banach space theory. In the first part of this work, we will associate to each Baire class 1 function on $E$ three different ordinal ranks, each corresponding to one of the standard equivalent descriptions of Baire class 1 functions, in terms of (i) complexity of inverse images of open sets, (ii) existence of continuity points on closed sets, and (iii) as limits of sequences of continuous functions. We will then show the essential equivalence of these ranks for bounded Baire class 1 functions, thus establishing a "quantitative" version of the equivalence of the above descriptions.

This yields, in $\S 2$, a natural classification of the set $\mathscr{B}_{1}(E)$ of bounded Baire class 1 functions into a transfinite hierarchy of Banach algebras $\mathscr{B}_{1}^{\xi}(E)$, $\xi<\omega_{1}$, that we call (bounded) functions of small Baire class $\xi$. In $\S 3$, we study the first level $\mathscr{B}_{1}^{1}$ of this hierarchy, showing that it corresponds to a previously considered notion, the so-called strict Baire class 1 functions, the uniform limits of differences of bounded semicontinuous functions. We also define a natural rank on the subset DBSC of differences of bounded semicontinuous functions, and a rank on functions not in DBSC, and show that these two ranks are unbounded below $\omega_{1}$, on DBSC and on $\mathscr{B}_{1}{ }^{1} \backslash$ DBSC respectively.

In $\S 4$, we give two other different approaches to our classification. The first one uses the notion of (transfinite) alternating sums of usc functions, and allows

Received by the editors December 15, 1987 and, in revised form, June 3, 1988.

1980 Mathematics Subject Classification (1985 Revision). Primary 03E15, 04A15, 46B99.

Research of the first author was partially supported by NSF Grant DMS-8718847. 
us to characterize each level $\mathscr{B}_{1}^{\xi}$ in a way very similar to the classical HausdorffKuratowski analysis of $\Delta_{2}^{0}$ as differences of closed sets.

The second one uses a notion of pseudouniform convergence of a sequence of functions, intermediate between uniform and pointwise convergence. Starting from the continuous functions on $E$ and closing under the taking of pseudouniform limits of bounded sequences yields the bounded Baire class 1 functions on $E$. Moreover, the natural hierarchy within $\mathscr{B}_{1}$ associated to this operation is exactly the hierarchy $\left(\mathscr{B}_{1}^{\xi}\right)_{\xi<\omega_{1}}$.

In the final section, we come back to one of the tools we introduce for studying Baire class 1 functions, the notion of optimally converging sequence, which generalizes the concept of uniform convergence for the case where $\lim f_{n}=f$ is not continuous. Roughly speaking, a sequence $\left(f_{n}\right)$ of continuous functions optimally converges to $f$ if it converges (pointwise) to $f$, and its rate of convergence is least among all sequences of continuous functions converging to it (this is measured by a countable ordinal, see $\S 1$ ). Extending the classical result about uniform convergence, we show in $\S 1$ that for any bounded sequence of continuous functions converging to some $f$, there exists a sequence of convex combinations optimally converging to $f$.

Applying this to the case of derivatives, we show that for any differentiable function $F$ on, say, $[0,1]$ there is a sequence of step functions $h_{n}(x)$ uniformly converging to 0 in $n$ such that the associated differences

$$
\left(F\left(x+h_{n}(x)\right)-F(x)\right) / h_{n}(x)
$$

optimally converge to the derivative $F^{\prime}$, but we construct examples of differentiable functions $F$ for which for any sequence of numbers $h_{n} \rightarrow 0$, the sequence $\left(F\left(x+h_{n}(x)\right)-F(x)\right) / h_{n}$ is arbitrarily far from being optimal.

\section{THE THREE RANKS}

Here and below $E$ is a compact, metrizable space.

I. The separation rank (Bourgain [B]). Let $A, B$ be two subsets of $E$. We associate with them a derivation on closed sets, by

$$
P_{A, B}^{\prime}=\overline{P \cap A} \cap \overline{P \cap B}
$$

and then by transfinite induction $P_{A, B}^{(0)}=P, P_{A, B}^{(\alpha+1)}=\left(P_{A, B}^{(\alpha)}\right)_{A, B}^{\prime}$ and $P_{A, B}^{(\lambda)}=$ $\bigcap_{\kappa<\lambda} P_{A, B}^{(\alpha)}$ for limit $\lambda$. We set

$$
\alpha(P, A, B)=\left\{\begin{array}{l}
\text { least } \alpha\left(P_{A, B}^{(\alpha)}\right)=\varnothing, \quad \text { if such an } \alpha \text { exists } \\
\omega_{1} \text { otherwise }
\end{array}\right.
$$

and let $\alpha(A, B)=\alpha(E, A, B)$. As is well known, $\alpha(A, B)<\omega_{1}$ iff one can separate $A$ from $B$ by a set which is a (transfinite) difference of closed sets, and the ordinal $\alpha(A, B)$ "measures" the minimal length of such a difference. (It is not hard to check that if $\alpha(A, B)=\lambda+n$, with limit $\lambda$, then the minimal length 
is either $\lambda+2 n$ or $\lambda+2 n-1$. Note that classically one uses a slight variant, the derivation $P_{A, B}^{*}=\overline{\overline{P \cap A} \cap B}$ which is not symmetric, and less adapted to our purposes.)

Suppose now $f: E \rightarrow \mathbb{R}$. For each pair $r_{1}<r_{2}$ of rationals, let

$$
\alpha\left(f, r_{1}, r_{2}\right)=\alpha\left(\left\{x \in E: f(x) \leq r_{1}\right\},\left\{x \in E: f(x) \geq r_{2}\right\}\right)
$$

and finally define the separation rank $\alpha(f)$ by

$$
\alpha(f)=\sup \left\{\alpha\left(f, r_{1}, r_{2}\right): r_{1} \in \mathbb{Q}, r_{2} \in \mathbb{Q}, r_{1}<r_{2}\right\} .
$$

Proposition 1. A function $f$ is Baire class 1 iff $\alpha(f)<\omega_{1}$.

Proof. If $\alpha(f)<\omega_{1}$, one can always separate $\{f \leq a\}$ from $\{f \geq b\}$, for $a<b$, by a $G_{\delta} \cap F_{\sigma}$ set by the definition of $\alpha(f)$. Hence $\{f \leq a\}$ is an intersection of countably many $G_{\delta}$ sets, hence is $G_{\delta}$, and similarly for $\{f \geq a\}$. It easily follows that $f^{-1}(F)$ is $G_{\delta}$ for all closed $F$ in $\mathbb{R}$, i.e., $f$ is Baire class 1. Conversely, if $f$ is Baire class $1, A=\left\{f \leq r_{1}\right\}$ and $B=\left\{f \geq r_{2}\right\}$ are disjoint $G_{\delta}$. sets in $E$, and as is well known, this implies $\alpha\left(f, r_{1}, r_{2}\right)<\omega_{1}$. (Note that if $P=E_{A, B}^{\omega_{1}} \neq \varnothing, P=\overline{P \cap A} \cap \overline{P \cap B}$, so that $P \cap A$ and $P \cap B$ are both dense $G_{\delta}$ sets in $P$, hence $P \cap A \cap B \neq \varnothing$, a contradiction.) And finally $\alpha(f)=\sup _{r_{1}<r_{2}} \alpha\left(f, r_{1}, r_{2}\right)$ is less then $\omega_{1}$ too.

This rank was introduced by Bourgain in [B], who showed that if $\left(f_{n}\right)$ is a bounded sequence of continuous functions on $E$ which is relatively compact (for the pointwise convergence) in $\mathscr{B}_{1}(E)$, then the rank $\alpha$ is uniformly bounded on the closure of $\left(f_{n}\right)$.

However, this rank does not seem very convenient if we consider functions which are not necessarily bounded. We will see, e.g., that $\sup \{\alpha(f+g): f$, $g$ Baire class 1 with $\alpha(f)=\alpha(g)=2\}=\omega_{1}$.

II. The oscillation rank. (This rank has been considered by many authors, including S. Argyros, R. Haydon and others, see e.g. [H-O-R].)

Define, for $f: E \rightarrow \mathbb{R}$, the oscillation function

$$
\operatorname{osc}(f, x)=\inf \left\{\sup _{x_{1}, x_{2} \in V}\left|f\left(x_{1}\right)-f\left(x_{2}\right)\right|: V \text { open, } X \in V\right\}
$$

and similarly, for $P$ a subset of $E$,

$$
\operatorname{osc}(f, x, P)=\operatorname{osc}\left(\left.f\right|_{P}, x\right) \text {. }
$$

Consider now, for each $\varepsilon>0$, the derivative operation

$$
P \mapsto P_{\varepsilon, f}^{*}=\{x \in P: \omega(f, x, P) \geq \varepsilon\}
$$

and by iterating define again $P_{\varepsilon, f}^{\alpha}$ for $\alpha<\omega_{1}$, and let

$$
\beta(f, \varepsilon, P)=\left\{\begin{array}{l}
\text { least } \alpha\left(P_{\varepsilon, f}^{\alpha}=\varnothing\right), \quad \text { if such an } \alpha \text { exists, } \\
\omega_{1} \text { otherwise }
\end{array}\right.
$$

and set $\beta(f, \varepsilon)=\beta(f, \varepsilon, E)$ and finally define the oscillation rank $\beta(f)$ of $f$ by $\beta(f)=\sup _{\varepsilon>0} \beta(f, \varepsilon)$. 
Proposition 2. A function $f: E \rightarrow \mathbb{R}$ is Baire class 1 iff $\beta(f)<\omega_{1}$.

Proof. We use the following well-known characterization of Baire class 1 functions: $f$ is Baire class 1 iff for every nonempty closed $P \operatorname{osc}(f, P)$ takes arbitrary small values, i.e., for all $\varepsilon>0 \quad P_{\varepsilon, f}^{*} \neq P$. It follows that if $f$ is Baire class 1 , and $\varepsilon>0$, the sequence $E_{\varepsilon, f}^{\alpha}$ must stabilize at $\varnothing$, i.e., $\beta(f, \varepsilon)<\omega_{1}$. Conversely, if $f$ is not Baire class 1 , then for some nonempty closed $P$ and some $\varepsilon>0, P_{\varepsilon, f}^{*}=P$, and by induction $P \subseteq E_{\varepsilon, f}^{\alpha}$ for all $\alpha<\omega_{1}$, hence $\beta(f, \varepsilon)=\omega_{1}$.

III. The convergence rank. Given a sequence $\bar{f}=\left(f_{n}\right)$ of real functions on $E$, define, following Zalcwasser [Z] and Gillespie and Hurwicz [G-H] a derivative operation on closed sets, for each $\varepsilon>0$ by

$$
\begin{array}{r}
P \mapsto P_{\varepsilon, \bar{f}}^{\prime}=\{x \in P \text { : for every nbhd } U \text { of } x \text { and any } p \in \mathbb{N}, \exists n>m>p \\
\text { and } \left.x^{\prime} \in P \cap U \text { with }\left|f_{n}\left(x^{\prime}\right)-f_{m}\left(x^{\prime}\right)\right| \geq \varepsilon\right\} .
\end{array}
$$

(In other words, $P_{\varepsilon, \bar{f}}^{\prime}$ is obtained from $P$ by deleting the relatively open sets in $P$ on which the sequence $\left(f_{n}\right)$ is $\varepsilon$-uniformly convergent.)

Again define by induction the iterates $P_{\varepsilon, \bar{f}}^{\alpha}$, and let

$$
\gamma(\bar{f}, \varepsilon, P)=\left\{\begin{array}{l}
\text { least } \alpha\left(P_{\varepsilon, \bar{f}}^{\alpha}=\varnothing\right), \quad \text { if such an } \alpha \text { exists } \\
\omega_{1} \text { otherwise },
\end{array}\right.
$$

and also $\gamma(\bar{f}, \varepsilon)=\gamma(\bar{f}, \varepsilon, E)$ and $\gamma(\bar{f})=\sup _{\varepsilon>0} \gamma(\bar{f}, \varepsilon)$.

It is easy to verify that if $\bar{f}$ is a sequence of functions on $E$ with $\gamma(\bar{f})<\omega_{1}$, the sequence $\bar{f}$ pointwise converges on $E$. For if $x \in E$ and $\varepsilon>0$ is given, $x \notin E_{\varepsilon, \bar{f}}^{\alpha}$ for some $\alpha$, hence in particular $\exists n_{0}, \forall n, m \geq n_{0}\left|f_{n}(x)-f_{m}(x)\right|<\varepsilon$ and the sequence $\left(f_{n}(x)\right)$ is Cauchy in $\mathbb{R}$. A sequence $\left(f_{n}\right)$ with $\gamma\left(f_{n}\right)<\omega_{1}$ is sometimes called quasiuniformly convergent. Note that if $\bar{f}$ consists of Baire class 1 functions and $\gamma(\bar{f})<\omega_{1}$, the pointwise limit $f=\lim _{n} f_{n}$ is also Baire class 1 , so that in general pointwise convergence does not imply quasiuniform convergence. However the notions coincide for sequences of continuous functions:

Proposition 3. Let $\bar{f}=\left(f_{n}\right)$ be a sequence of continuous functions on $E$, pointwise converging to some $f$. Then $\gamma(\bar{f})<\omega_{1}$.

Proof. It is enough to show that $P \neq \varnothing$ and $\varepsilon>0, P_{\varepsilon, \bar{f}}^{\prime} \neq P$. Assume the contrary, so that for each $p \in \mathbb{N},\left\{x \in P: \exists m, n \geq p\left|f_{m}(x)-f_{n}(x)\right|>\varepsilon / 2\right\}$ is dense, and clearly open, in $P$. By Baire's theorem, there is an $x \in P$ with $\forall p$ $\exists m, n \geq p\left|f_{n}(x)-f_{n}(x)\right|>\varepsilon / 2$, and the sequence $\left(f_{n}(x)\right)$ does not converge.

It is immediate to check that $\bar{f}$ converges uniformly iff $\gamma(\bar{f})=1$. (Here compactness of $E$ is used in an essential way.) In $\S 4$, we will also consider an intermediate convergence notion, corresponding to $\gamma(\bar{f}) \leq \omega$. 
We now come back to ranks of Baire class 1 functions. As is well known, Baire class 1 functions on $E$ are pointwise limits of sequences of continuous functions; hence, we can define the convergence rank $\gamma(f)$ of a Baire class 1 function $f$ on $E$ by

$$
\gamma(f)=\inf \left\{\gamma(\bar{f}): \bar{f} \in C(E)^{\mathbb{N}}, \bar{f} \rightarrow f \text { pointwise }\right\},
$$

so that the ordinal $\gamma(f)$ measures the "best possible rate" of convergence to $f$ of a sequence of continuous functions. And we say that a sequence $\bar{f}=\left(f_{n}\right)$ of continuous functions is optimally convergent if it pointwise converges to some $f$, and moreover $\gamma(f)=\gamma(\bar{f})$. Heuristically, this notion is interesting among other things in concrete situations in analysis, when the Baire class 1 functions occur through a "natural" approximation process, like in the cases of derivatives and Fourier series. The question whether the natural approximation process is always optimal (in the above precise sense) may be a clue about the difficulty of the problems under study. We will come back to this question in the last section, with the particular case of derivatives.

\section{THE HIERARChY $\left(\mathscr{B}_{1}^{\xi}\right)_{\xi<\omega_{1}}$ OF (BOUNDED) SMALL BAIRE CLASS $\xi$ FUNCTIONS}

Our main purpose in this section is to relate the various ranks defined in $\S 1$. First an easy proposition.

Proposition 1. Let $f$ be a Baire class 1 function on $E$. Then $\alpha(f) \leq \beta(f) \leq$ $\gamma(f)$.

Proof. (i) $\alpha(f) \leq \beta(f)$. Let $r_{1}<r_{2}$ be given in $\mathbb{Q}, A=\left\{f \leq r_{1}\right\}$ and $B=\left\{f \geq r_{2}\right\}$. Let $\varepsilon=r_{2}-r_{1}$. If $P$ is closed and $x \in P \backslash P_{\varepsilon, f}^{*}$, there exists a nbhd $V$ of $x$ such that $\operatorname{osc}(f, V \cap P)<\varepsilon$, so that $V$ cannot meet both $A \cap P$ and $B \cap P$. This $P_{A, B}^{\prime} \subseteq P_{\varepsilon, f}^{*}$ for all $P$, and hence by induction $P_{A, B}^{\alpha} \subseteq P_{\varepsilon, f}^{\alpha}$ and $\alpha\left(f, r_{1}, r_{2}\right) \leq \beta\left(f, r_{2}-r_{1}\right)$ so that $\alpha(f) \leq \beta(f)$.

(ii) $\beta(f) \leq \gamma(f)$. Let $\bar{f}$ be a sequence of continuous functions with $\lim \bar{f}=$ $f$. We want $\beta(f) \leq \gamma(\bar{f})$. As before it is enough to show, for any closed set $P$ and $\varepsilon>0, P_{\varepsilon, f}^{*} \subseteq P_{\varepsilon / 3, \bar{f}}^{\prime}$. But if $x \in P \backslash P_{\varepsilon / 3, \bar{f}}^{\prime}$, there is a nbhd $V$ of $x$ and $n_{0} \in \mathbb{N}$ with $\left|f_{n}\left(x^{\prime}\right)-f_{m}\left(x^{\prime}\right)\right|<\varepsilon / 3$ for $m, n \geq n_{0}$ and $x^{\prime} \in P \cap V$. Letting $m \rightarrow \infty$ we get $\left|f_{n}\left(x^{\prime}\right)-f\left(x^{\prime}\right)\right| \leq \varepsilon / 3$ for all $n \geq n_{0}$ and $x^{\prime} \in P \cap V$. Let $V^{\prime} \subseteq V$ be a nbhd of $x$ where $\operatorname{osc}\left(f_{n_{0}}\right)<\varepsilon / 3$. Then on $V^{\prime} \cap P$

$$
\left|f\left(x^{\prime}\right)-f\left(x^{\prime \prime}\right)\right| \leq\left|f_{n_{0}}\left(x^{\prime}\right)-f_{n_{0}}\left(x^{\prime \prime}\right)\right|+2 \varepsilon / 3<\varepsilon .
$$

Proposition 2. Let $f$ be a Baire class 1 function. Then

(i) $f$ is continuous on $E$ iff $\alpha(f)=\beta(f)=\gamma(f)=1$.

(ii) If $f$ is semicontinuous on $E, \alpha(f) \leq 2$.

(iii) There exists on $E=[0,1]$ a bounded usc function $f$ with $\beta(f)=\omega$. 
Proof. (i) If $f$ is continuous, $\gamma(f)=1$ by considering the constant sequence $\bar{f}=\left(f_{n}\right)$ with $f_{n}=f$. If $\alpha(f)=1,\left\{\overline{f \leq r_{1}}\right\} \cap\left\{\overline{f \geq r_{2}}\right\}=\varnothing$ for all $r_{1}<r_{2}$ in $\mathbb{Q}$, hence $\left\{f \leq r_{1}\right\}$ and $\left\{f \geq r_{2}\right\}$ are closed for all $r_{1}, r_{2}$, and finally $f^{-1}(F)$ is closed for any closed set $F \subseteq \mathbb{R}$.

(ii) If $f$ is (say) usc, then $B=\left\{f \geq r_{2}\right\}$ is closed, hence if $A=\left\{f \leq r_{1}\right\}$,

$$
P_{A, B}^{\prime}=\overline{P \cap A} \cap \overline{P \cap B}=\overline{P \cap A} \cap B \subseteq B,
$$

hence $P_{A, B}^{\prime \prime} \subseteq \overline{A \cap B}=\varnothing$ for any closed $P$, and $\alpha(f) \leq 2$.

(iii) Let $K_{n} \subseteq[0,1]$ be a decreasing sequence of nonempty compact sets, with $K_{n+1}$ nowhere dense in $K_{n}$. Let $f=\sum_{n} 2^{-n} \cdot 1_{K_{n}}$. Clearly $f$ is usc, with $0 \leq f \leq 1$ on $E$. Now a direct computation shows that for $\varepsilon=2^{-n}$, $E_{\varepsilon, f}^{p}=K_{p}$ for $1 \leq p \leq n$ and $E_{\varepsilon, f}^{n+1}=\varnothing$, so that $\beta\left(f, 2^{-n}\right)=n+1$ and $\boldsymbol{\beta}(f)=\omega$.

The preceding example shows that one cannot hope equality between the ranks. We will see that $\omega$ is the upper bound of $\beta(f)$ (and $\gamma(f)$ ), for bounded functions $f$ with $\alpha(f)=2$, and that there is no upper bound in case $f$ is allowed to be unbounded.

The next result gives the exact relationship between $\beta(f)$ and $\gamma(f)$ in case $f$ is bounded, in a very strong form.

If $\bar{f}_{-}=\left(f_{n}\right)$ is a sequence of functions, let us say that a sequence $\bar{g}=\left(g_{n}\right)$ is subordinate to $\left(f_{n}\right)$, denoted $\bar{g} \prec \bar{f}$, if for all $n \quad g_{n} \in \operatorname{conv}\left(\left(f_{p}\right)_{p \geq n}\right)$. (Here $\operatorname{conv}\left(\left(h_{k}\right)\right)$ denotes the set of convex combinations of the $h_{k}$ 's.)

A classical result of Mazur asserts that if $\bar{f}=\left(f_{n}\right)$ is a bounded sequence of continuous functions on $E$ which pointwise converges to a continuous function $f$, there exists a sequence $\bar{g}$ subordinated to $\bar{f}$ which uniformly converges to $f$. The following result is the generalization of Mazur's result to the case where $f$ is not necessarily continuous.

Theorem 3. Let $\bar{f}=\left(f_{n}\right)$ be a bounded sequence of continuous functions on $E$, pointwise converging to some (bounded) Baire class 1 function $f$. Then there exists a sequence $\bar{g}$ subordinated to $\bar{f}$ with $\gamma(\bar{g})=\beta(f)$. In particular, for any bounded Baire class 1 function $f$, the convergence rank $\gamma(f)$ and the oscillation rank $\beta(f)$ coincide. (To see that this is indeed a generalization, notice that if $f$ is continuous, then $\beta(f)=1$, so $\gamma(\bar{g})=1$, i.e., $\bar{g}$ converges uniformly.)

Proof. Note the following easy application of the Hahn-Banach theorem: If $K \subseteq E$ is compact and $\left(f_{n}\right)$ is a bounded sequence in $C(K)$ converging pointwise to $f$, and $\operatorname{osc}(f, K)<\varepsilon$, then $\exists g \in \operatorname{conv}\left(\left(f_{n}\right)\right)\|g-f\| \leq \varepsilon$. (Here $\left.\|h\|=\|h\|_{K}=\sup _{x \in K}|h(x)|.\right)$

[To see this, let $\operatorname{osc}(f, K)=2 \varepsilon^{\prime}<\varepsilon$. By adding a constant if necessary, we may assume $\|f\|=\varepsilon^{\prime}$, and it is enough to find $g \in A=\overline{\operatorname{conv}}\left(\left(f_{n}\right)\right)$ with $\|g\|<\delta=\varepsilon-\varepsilon^{\prime}$. If $A$ and $B_{\delta}=\{g \in C(K):\|g\|<\delta\}$ are disjoint, we can by Hahn-Banach find a measure $\mu \in M(K)$ with, for some $c>0, \mu(h)<c \leq \mu(g)$ 
for $h \in B_{\delta}$ and $g \in A$. The first inequality gives $\|\mu\| \leq c / \delta$. The second one gives $\mu\left(f_{n}\right) \geq c$ for all $n$, and by bounded convergence $\mu(f) \geq c$. But then $c \leq \mu(f) \leq\|\mu\|\|f\|<c \cdot \varepsilon^{\prime} / \delta$, i.e., $\varepsilon^{\prime} \geq \delta=\varepsilon-\varepsilon^{\prime}$ contradicting the fact that $\left.2 \varepsilon^{\prime}<\varepsilon.\right]$

Applying this fact to each $A_{p}=\operatorname{conv}\left(\left(f_{n}\right)_{n \geq p}\right)$, one gets that if $\left(f_{n}\right)$ is a bounded sequence in $C(K)$ converging pointwise to $f$ on $K$ with $\operatorname{osc}(f, K)<$ $\varepsilon$, there is a subordinated $\bar{g}=\left(g_{n}\right) \prec\left(f_{n}\right)$ with $\left\|g_{n}-f\right\|_{K} \leq \varepsilon$ for all $n$.

Let now $\left(f_{n}\right)$ be bounded in $C(E), f_{n} \rightarrow f$. Let $\left(U_{m}\right)_{m \in \mathbb{N}}$ be a basis for $E$, and enumerate in a single sequence $\left(K_{p}, \varepsilon_{p}\right)$ all pairs of form $\left(E_{2^{-k}, f}^{\xi} \cap \bar{U}_{m}, 2^{-k}\right)$ where $\xi<\beta\left(f, 2^{-k}\right)$, and $m$ is such that $E_{2^{-k}, f}^{\xi} \cap U_{m} \neq$ $\varnothing$ and $\operatorname{osc}\left(f, E_{2-k, f}^{\xi} \cap U_{m}\right)<2^{-k}$. Applying the above remarks successively to each $K_{p}$ and $\varepsilon_{p}$, one defines inductively $\bar{g}^{p}$ such that $\bar{g}_{0}=\bar{f}$ and $\bar{g}^{p+1} \prec$ $\bar{g}^{p}$, with $\left\|g_{n}^{p}-f\right\|_{K_{p}} \leq \varepsilon_{p}$ for all $n$. Let $\bar{g}=\left(g_{n}\right)$ be the diagonal sequence, i.e., $g_{n}=g_{n}^{n}$. Clearly $\bar{g} \prec \bar{f}$. Moreover for all $p\left\|g_{n}-f\right\|_{K_{p}} \leq \varepsilon_{p}$ for $n \geq p$, because $\left\|g_{q}^{p}-f\right\|_{K_{p}} \leq \varepsilon_{p}$ for all $q$, and for $n \geq p \quad g_{n}=g_{n}^{n} \in \operatorname{conv}\left(\left(g_{q}^{p}\right)_{q}\right)$.

We claim that $\gamma(\bar{g}) \leq \beta(f)$. To see this, it is enough to show that for $\varepsilon>0$ and $k$ such that $2^{-k}<\varepsilon / 2$, one has for all $\xi<\omega_{1} E_{\varepsilon, \bar{g}}^{\xi} \subseteq E_{2^{-k}, f}^{\xi}$. This is proved by induction on $\xi$. The case $\xi=0$ and $\xi$ limit are trivial. So assuming $E_{\varepsilon, \bar{g}}^{\xi} \subseteq E_{2^{-k}, f}^{\xi}$, we show that $E_{\varepsilon, \bar{g}}^{\xi+1} \subseteq E_{2^{-k}, f}^{\xi+1}$. Let $x \in E_{\varepsilon, \bar{g}}^{\xi} \backslash E_{2^{-k}, f}^{\xi+1}$. Then for some $m x \dot{x} \in U_{m}$ and $K=E_{2^{-k}, f}^{\xi} \cap \bar{U}_{m}$ is such that $\operatorname{osc}(f, K)<2^{-k}$. Then for some $p\left(K, 2^{-k}\right)=\left(K_{p}, \varepsilon_{p}\right)$, so that for $n \geq p, m \geq p\left\|g_{n}-g_{m}\right\|_{K}<$ $2 \cdot\left\|g_{n}-f\right\|_{K} \leq 2 \varepsilon_{p}<\varepsilon$ and a fortiori this is true on $\bar{U}_{m} \cap E_{\varepsilon, \bar{g}}^{\xi} \subseteq K \cap E_{\varepsilon, \bar{g}}^{\xi}$, so that $x \notin E_{\varepsilon, \bar{g}}^{\xi+1}$. This finishes the proof.

The preceding result has lots of applications besides proving $\beta(f)=\gamma(f)$ for bounded Baire class 1 functions. It can be used, for example, to show that many properties of a bounded Baire class 1 function $f$ are shared by sequences of continuous functions optimally converging to it. Here are some instances of this phenomenon:

1. If $\|f\| \leq M$, there is $f_{n} \rightarrow f$ optimally with $\sup _{n}\left\|f_{n}\right\| \leq M$.

2. If $E$ is convex compact metrizable, and $f$ is affine Baire class 1 , there is a sequence of affine continuous $f_{n}$ 's converging optimally to $f$.

3. If $X$ is a separable Banach space, $E=B_{1}\left(X^{*}\right)$ is the unit ball of the dual $X^{*}$ with the $w^{*}$-topology, and $x^{* *}$ is an element of $X^{* *}$ which is first class on $E$, there is a sequence $\left(x_{n}\right) \in X$ converging optimally to $x^{* *}$ on $E$.

To see this, it is enough by the preceding theorem to show that in each case there exists a bounded sequence $\left(f_{n}\right)$ of continuous functions with the desired properties converging pointwise to $f$, for the properties clearly go to subordinated sequences. This is trivial for (1), and due to Choquet [C] for (2), and to Odell and Rosenthal [O-R] for (3). 
It is a standard fact that a decreasing sequence of continuous functions which converges to a continuous function converges uniformly (Dini's Theorem). Using Theorem 3 this can be generalized as follows: If $\left(f_{n}\right)$ is a bounded decreasing sequence of continuous functions, $\left(f_{n}\right)$ converges optimally. (To see that this generalizes Dini's Theorem notice that if $f_{n} \rightarrow f$ optimally and $f$ is continuous, then $f_{n} \rightarrow f$ uniformly.)

To prove this result notice that if $h_{n} \leq g_{n}$ and $g_{n} \downarrow f, h_{n} \downarrow f$, then $\gamma(\bar{h}) \leq$ $\gamma(\bar{g})$. Also if $h_{n} \downarrow f$ and $\left(h_{k_{n}}\right)$ is a subsequence of $\left(h_{n}\right)$ then $\gamma\left(\left(h_{k_{n}}\right)\right)=\gamma(\bar{h})$. So assume $\bar{f}$ is bounded and $f_{n} \downarrow f$. We will show that $\gamma(\bar{f})=\gamma(f)$. By Theorem 3 let $\bar{g}$ subordinated to $\bar{f}$ be such that $\gamma(\bar{g})=\gamma(f)$. Then there is a subsequence $\left(f_{k_{n}}\right)$ of $\left(f_{n}\right)$ such that $f_{k_{n}} \leq g_{n}$. So $\gamma(\bar{f}) \leq \gamma\left(\left(f_{k_{n}}\right)\right) \leq \gamma(\bar{g})=$ $\gamma(f)$, i.e., $\bar{f}$ converges optimally.

Another application is the following - the first step in relating the ordinals $\alpha(f)$ and $\beta(f)$.

Corollary 4. Let $A \in \Delta_{2}^{0}=F_{\sigma} \cap G_{\delta}$ (i.e., $1_{A}$ is Baire class 1). Then $\alpha\left(1_{A}\right)=$ $\beta\left(1_{A}\right)=\gamma\left(1_{A}\right)=\alpha(A, \check{A})$ (where $\check{A}=E \backslash A$ is the complement of $A$ ).

Proof. $\alpha(A, \check{A})=\alpha\left(1_{A}, 1 / 2\right) \leq \alpha\left(1_{A}\right) \leq \beta\left(1_{A}\right)=\gamma\left(1_{A}\right)$ where the last equality follows from Theorem 3 . So it remains to show $\beta\left(1_{A}\right) \leq \alpha(A, \breve{A})$. But clearly for any $\varepsilon$ with $0<\varepsilon<1$, and $P$ closed in $E \quad P_{\varepsilon, 1_{A}}^{*}=\overline{P \cap A} \cap \overline{P \cap \check{A}}$ and we are done.

We now turn to the relationship between $\alpha$ and $\gamma$.

Lemma 5. Let $\bar{f}=\left(f_{n}\right)$ and $\bar{g}=\left(g_{n}\right)$ be two sequences of functions, pointwise converging to $f$ and $g$ respectively. Set $\bar{f}+\bar{g}=\left(f_{n}+g_{n}\right)_{n \in \mathbb{N}}$. If $\xi<\omega_{1}$ is such that $\gamma(\bar{f}) \leq \omega^{\xi}$ and $\gamma(\bar{g}) \leq \omega^{\xi}$ then $\gamma(\bar{f}+\bar{g}) \leq \omega^{\xi}$.

Proof. We first establish the following two facts:

(i) Let $P$ be closed, $\varepsilon>0$. Then

$$
P_{\varepsilon, \bar{f}+\bar{g}}^{\prime} \subseteq P_{\varepsilon / 2, \bar{f}}^{\prime} \cup P_{\varepsilon / 2 \bar{g}}^{\prime} .
$$

(ii) Let $P, Q$ be closed, $\varepsilon>0$. Then

$$
(P \cap Q)_{\varepsilon, \bar{f}}^{\prime} \subseteq P_{\varepsilon, \bar{f}}^{\prime} \cup Q_{\varepsilon, \bar{f}}^{\prime} .
$$

Fact (i) is immediate: If $x \in P \backslash\left(P_{\varepsilon / 2, \bar{f}}^{\prime} \cup P_{\varepsilon / 2, \bar{g}}^{\prime}\right)$ there exists $V$ nbhd of $x$ and $n_{0}$ (we can take the same for both) such that for $n, m \geq n_{0}\left|f_{n}-f_{m}\right|<\varepsilon / 2$ on $V \cap P$ and $\left|g_{n}-g_{m}\right|<\varepsilon / 2$ on $V \cap P$, so $\left|f_{n}+g_{n}-\left(f_{m}+g_{m}\right)\right|<\varepsilon$ on $V \cap P$ and $x \notin P_{\varepsilon, \bar{f}+\bar{g}}^{\prime}$. Fact 2 is similar: If $x \in P \cup Q$ is not in $P_{\varepsilon, \bar{f}}^{\prime} \cup Q_{\varepsilon, \bar{f}}^{\prime}$, there is a nbhd $V$ of $x$ and an $n_{0}$ (we can take the same for both) such that for $m, n \geq n_{0}\left|f_{m}-f_{n}\right|<\varepsilon$ on $V \cap P$ and $\left|f_{m}-f_{n}\right|<\varepsilon$ on $V \cap Q$. But then $\left|f_{m}-f_{n}\right|<\varepsilon$ on $V \cap(P \cup Q)$ and $x \notin(P \cup Q)_{\varepsilon, \bar{f}}^{\prime}$.

We now prove, by induction on $\xi$, that

$$
P_{\varepsilon, \bar{f}+\bar{g}}^{\omega_{\xi}^{\xi}} \subseteq P_{\varepsilon / 2, \bar{f}}^{\omega^{\xi}} \cup P_{\varepsilon / 2, \bar{g}}^{\omega^{\xi}}
$$

which will finish the proof. 
For $\xi=0$, i.e., $\omega^{\xi}=1$, this is just $(*)$. It is immediate if $\xi$ is limit, as the sequences are decreasing. So it remains to prove that $(* * *)$ holds for $\xi+1$ if it holds for $\xi$. For this, we prove for all $n \geq 1$ that

$$
P_{\varepsilon, \bar{f}+\bar{g}}^{\omega^{\xi} \cdot 2 n} \subseteq P_{\varepsilon / 2, \bar{f}}^{\omega^{\xi} \cdot n} \cup P_{\varepsilon / 2, \bar{g}}^{\omega^{\xi} \cdot n}
$$

For this, let for $s \in 2^{k}, k \in \mathbb{N}, P_{s}$ be defined by

$$
P_{0}=P_{\varepsilon / 2, \bar{f}}^{\omega^{\xi}}, \quad P_{1}=P_{\varepsilon / 2, \bar{g}}^{\omega^{\xi}}
$$

and $P_{s^{\wedge} 0}=\left(P_{s}\right)_{\varepsilon / 2, \bar{f}}^{\omega^{\xi}}, P_{s^{\wedge} 1}=\left(P_{s}\right)_{\varepsilon / 2, \bar{g}}^{\omega^{\xi}}$ for $\ln (s)>1$. Applying $\left(\omega^{\xi}\right.$ times $)$ $(* *)$ and the inclusion

$$
P_{\varepsilon, \bar{f}+\bar{g}}^{\omega^{\xi}} \subseteq P_{0} \cup P_{1}
$$

one easily gets for all $n \geq 1$

$$
\begin{aligned}
P_{\varepsilon, \bar{f}+\bar{g}}^{\omega^{\xi} \cdot 2 n} \subseteq & \bigcup \\
s \in 2^{2 n} & P_{s} \subseteq \bigcup\left\{P_{s}: s \in 2^{2 n} \text { and } \operatorname{card}(\{k: s(k)=0\}) \geq n\right\} \\
& \cup \bigcup\left\{P_{s}: s \in 2^{2 n} \text { and } \operatorname{card}(\{k: s(k)=1\}) \geq n\right\} .
\end{aligned}
$$

But clearly if $s$ takes at least $n$ values $0, P_{s} \subseteq P_{\varepsilon / 2, \frac{\omega^{k}}{\omega^{k}},}$, and similarly with 1 , which gives $(* * * *)$. And taking the intersection over $n$ gives $(* * *)$ for $\xi+1$. This finishes the proof.

Remark. The same argument gives the analogous result for $\bar{f} \cdot \bar{g}=\left(f_{n} \cdot g_{n}\right)_{n \in \mathbb{N}}$ in case $\bar{f}$ and $\bar{g}$ are bounded sequences, for if $\sup _{n}\left(\left\|f_{n}\right\|,\left\|g_{n}\right\|\right)=M$, one easily gets $P_{\varepsilon, \bar{f} \cdot \bar{g}}^{\prime} \subseteq P_{\varepsilon / 2 M, \bar{f}}^{\prime} \cup P_{\varepsilon / 2 M, \bar{g}}^{\prime}$.

Lemma 5 and Corollary 4 imply that in general, for unbounded Baire class 1 functions, the $\alpha$ and $\gamma$ ranks are not comparable. This is based on the following classical facts.

(1) If $E$ is uncountable, there are $\Delta_{2}^{0}$ sets $A$ with arbitrarily large small Baire class (i.e., $\alpha(A, \check{A})$ arbitrarily large ).

(2) If $A$ is $\Delta_{2}^{0}$ in $E, 1_{A}$ is a difference of two usc functions. [To see this, let $K_{n}$ be increasing closed sets with $A=\cup_{n} K_{n}, L_{n}$ increasing closed sets with $\breve{A}=\bigcup_{n} L_{n}$, and set

$$
f_{0}=\left\{\begin{array}{cl}
0 & \text { on } K_{0} \cup L_{0}, \\
-1 & \text { on }\left(K_{1} \cup L_{1}-K_{0} \cup L_{0}\right), \\
-n & \text { on }\left(K_{n} \cup L_{n}-\left(K_{n-1} \cup L_{n-1}\right)\right) \text { for } n \geq 1
\end{array}\right.
$$

and

$$
f_{1}=\left\{\begin{array}{cl}
0 & \text { on } L_{0}, \\
-1 & \text { on }\left(K_{0} \cup L_{1}\right)-L_{0}, \\
-n & \text { on }\left(K_{n-1} \cup L_{n}\right)-\left(K_{n-2} \cup L_{n-1}\right) \text { for } n \geq 2 .
\end{array}\right.
$$

Then each $f_{i}$ is usc, and $1_{A}=f_{0}-f_{1}$.] 
Proposition 6. Let $E$ be uncountable. Then

(i) $\sup \{\gamma(f): f$ usc on $E\}=\omega_{1}$,

(ii) $\left\{\alpha\left(f_{1}+f_{2}\right): \alpha\left(f_{1}\right) \leq 2, \alpha\left(f_{2}\right) \leq 2\right\}=\omega_{1}$.

Proof. For semicontinuous $f$ on $E, \alpha(f) \leq 2$, so part (ii) follows immediately from the preceding facts and Corollary 4.

If now $\{\gamma(f): f$ usc on $E\}$ was bounded below $\omega_{1}$, it would be bounded by some $\omega^{\xi}$, hence by Lemma 5 so would be $\left\{\gamma\left(f_{1}-f_{2}\right): f_{1}, f_{2}\right.$ usc on $\left.E\right\}$, which contains $\left\{\gamma\left(1_{A}\right): A \in \Delta_{2}^{0}\right\}$, contradicting again the facts above. This gives (i).

From now on, we restrict our attention to the space $\mathscr{B}_{1}(E)$ of bounded Baire class 1 functions on $E$.

Definition 7. Let $\xi$ be a countable ordinal. We define the set of (bounded) functions of small Baire class $\xi$ as

$$
\mathscr{B}_{1}^{\xi}(E)=\left\{f \in \mathscr{B}_{1}(E): \beta(f)(=\gamma(f)) \leq \omega^{\xi}\right\} .
$$

It is a Banach subalgebra of $\mathscr{B}_{1}(E)$, by Lemma 5 and the remark following it, and the immediate remark that if $f_{n} \rightarrow f$ uniformly, $\beta(f) \leq \sup _{n} \beta\left(f_{n}\right)$.

Theorem 8. Let $f \in \mathscr{B}_{1}(E)$. Then $f \in \mathscr{B}_{1}^{\xi}(E)$ iff $\alpha(f) \leq \omega^{\xi}$.

This result says in effect that the classical equivalences between the various possible descriptions of $\mathscr{B}_{1}$ functions reflect on each $\mathscr{B}_{1}^{\xi}$.

Proof. As $\alpha(f) \leq \beta(f)$, one implication is trivial. So we want to prove that if $\alpha(f) \leq \omega^{\xi}$, then $\gamma(f) \leq \omega^{\xi}$. But this is true if $f=1_{A}$ by Corollary 4 , if $f$ is a linear combination of $1_{A}$ 's with $\alpha\left(1_{A}\right) \leq \omega^{\xi}$ by Lemma 5 , and finally for all $f$ 's with $\alpha(f) \leq \omega^{\xi}$, using the following (essentially classical) result:

Proposition 9. Let $f \in \mathscr{B}_{1}(E)$, with $\alpha(f) \leq \omega^{\xi}$. Then $f$ is a uniform limit of linear combinations of functions $1_{A}$ with $\alpha\left(1_{A}\right)<\omega^{\xi}$. More precisely, if $f \in \mathscr{B}_{1}(E)$ with $\alpha(f) \leq \omega^{\xi}$ is positive, and $N>2, N \in \mathbb{N}$ is given, one can find $N-2$ sets $A_{1}, \ldots, A_{N-2}$ with $\alpha\left(1_{A_{k}}\right)<\omega^{\xi}$, such that the function $g=(\|f\| / N) \sum_{k=1}^{N-2} 1_{A_{k}}$ satisfies $0 \leq g \leq f \leq g+2\|f\| / N$.

Proof. The first assertion follows clearly from the second (which we will need in this precise form later on). So let $f \geq 0$ with $\alpha(f) \leq \omega^{\xi}$ and $N>2$ be given. By the assumption, there is for each $k^{k}=1, \ldots, N-2$ a $\Delta_{2}^{0}$ set $A_{k}$, with $\alpha\left(1_{A_{k}}\right)=\alpha\left(A_{k}, \check{A}_{k}\right) \leq \omega^{\xi}$, hence $<\omega^{\xi}$, as $\omega^{\xi}$ is limit, such that

$$
\left\{f \geq \frac{(k+1)\|f\|}{N}\right\} \subseteq A_{k} \subseteq\left\{f>\frac{k\|f\|}{N}\right\} .
$$

Let $g=(\|f\| / N) \sum_{k=1}^{N-2} 1_{A_{k}}$. We claim $g$ works: Clearly the sequence $A_{k}$ is decreasing, so $g$ is at most $k\|f\| / N$ off $A_{k+1}$. But $f>k\|f\| / N$ on $A_{k}$ for all 
$k$, and $f \geq 0$, so $g \leq f$. And $f<(k+1)\|f\| / N$ off $A_{k}$, whereas $g \geq k\|f\| / N$ on $A_{k}$, so $f \leq g+2\|f\| / N$.

Remark. Theorem 8 is in some sense best possible: For $E=[0,1]$ it can be shown that for any $\xi<\omega_{1}$ there are functions in $\mathscr{B}_{1}^{\xi+1}$ with $\alpha(f)=\omega^{\xi}+1$ and $\beta(f)=\omega^{\xi+1}$. We gave such an example for $\xi=0$ in Proposition 2. An example for arbitrary $\xi$ is as follows: Choose a decreasing sequence of closed sets $\left(F_{\eta}\right)_{\eta<\omega^{\xi+1}}$, with $F_{0}=E$ and for each $\eta, F_{\eta} \neq \varnothing$ and nowhere dense in $\bigcap_{\xi<\eta} F_{\xi}$, and let $f=\sum_{n} 2^{-n} \cdot 1_{A_{n}}$, where $A_{n}$ is the set difference of the sequence $\left(F_{\omega^{\xi} \cdot n+\theta}\right)_{\theta<\omega}$, i.e., $A_{n}=\bigcup\left\{F_{\omega^{\xi} \cdot n+\theta}-F_{\omega^{\xi} \cdot n+\theta+1}: \theta\right.$ even, $\left.\theta<\omega^{\xi}\right\}$, so that $0 \leq f \leq 1$. One easily checks that for all $r_{1}<r_{2}$ in $\mathbb{Q} \alpha\left(f, r_{1}, r_{2}\right) \leq \omega^{\xi}+1$ whereas for $\varepsilon=2^{-n}, \beta(f, \varepsilon)=\omega^{\xi} \cdot n+1$, the sequence $\left(F_{\eta}\right)_{1 \leq \eta \leq \omega^{\xi} \cdot n}$ being the sequence of derivatives. So $f$ works.

Theorem 8 says that the small class hierarchy $\left(\mathscr{B}_{1}^{\xi}\right)_{\xi<\omega_{1}}$ is quite natural. The level 0 consists exactly of the continuous functions. We will analyze $\mathscr{B}_{1}{ }_{1}^{1}$ in the next section. And we will give in $\S 4$ alternative approaches to the hierarchy $\left(\mathscr{B}_{1}^{\xi}\right)_{\xi<\omega_{1}}$ which give further indications of its naturalness.

\section{THE CLASS $\mathscr{B}_{1}^{1}$ AND DIFFERENCES OF BOUNDED SEMICONTINUOUS FUNCTIONS}

Let DSC be the set of differences of semicontinuous functions. We have seen that this set contains all $1_{A}, A \Delta_{2}^{0}$ in $E$, and by 2.9 we get that DSC is dense in $\mathscr{B}_{1}(E)$, for the norm topology. However there exist functions in $\mathscr{B}_{1}(E) \backslash \mathrm{DSC}$, by a result of $D$. Preiss (see [H-O-R]). Let now

$$
\text { DBSC }=\left\{f_{1}-f_{2}: f_{1}, f_{2} \text { semicontinuous and bounded on } E\right\} .
$$

As any bounded sc function has $\alpha(f) \leq 2$, hence $\gamma(f) \leq \omega$ by 2.8 , we get that $\mathrm{DBSC} \subseteq \mathscr{B}_{1}^{1}(E)$. In fact one has the following result, which appears in [H-O-R] (in slightly different terms).

Theorem 1. $\mathscr{B}_{1}^{1}(E)$ is the norm-closure of DBSC.

Proof. By 2.8, it is enough to show that any $1_{A}$ in $\mathscr{B}_{1}^{1}(E)$ is in fact in DBSC. But such a $1_{A}$ satisfies $\alpha(A, \breve{A})<\omega$, hence for some $n$ and decreasing closed sets $\left(F_{q}\right)_{0 \leq q \leq 2 n+1}$

$$
A=\bigcup_{p=0}^{n}\left(F_{2 p}-F_{2 p+1}\right) \text { and } 1_{A}=\left(\sum_{p} 1_{\cdot F_{2 p}}\right)-\left(\sum_{p} 1_{F_{2 p+1}}\right)
$$

in DBSC.

The functions in $\mathscr{B}_{1}^{1}(E)$ are sometimes called strict Baire class 1 functions, and $B_{1}^{1}(E)$ denoted $\mathscr{B}_{1 / 2}$ in [H-O-R] (a notation which can hardly extend in the transfinite!). This is related to the notion of strict sum, a different approach to the set DBSC: Say that $f$ is the strict sum of a sequence $f_{n}$ of functions if 
$f=\sum_{n}$, and $\sum_{n}\left|f_{n}\right|$ is a bounded function on $E$. It is not hard to check that the set of strict sums of continuous functions on $E$ is exactly DBSC: It clearly contains the bounded positive lsc functions, which are strict sums of positive continuous $f_{n}$ 's, and is a vector space, so contains DBSC. On the other hand, if $f=\sum_{n} f_{n}$, with $\sum_{n}\left|f_{n}\right|$ bounded, then $f=\sum f_{n}^{+}-\sum f_{n}^{-}$, and both sums are bounded lsc functions.

In order to clarify the relationship between DBSC and $\mathscr{B}_{1}{ }^{1}$, we now introduce two ranks, on DBSC and its complement, which might also be of independent interest.

Let $f$ be a bounded function on $E$. One defines the upper regularization of $f, \hat{f}$, by

$$
\begin{aligned}
\hat{f} & =\inf \{g: g \in C(E), g \geq f\} \\
& =\inf \{g: g \text { usc on } E, g \geq f\} .
\end{aligned}
$$

The function $\hat{f}$ is usc on $E$, and one has

$$
\hat{f}(x)=\inf \left\{\sup _{V}(f): V \text { nbhd of } x\right\} .
$$

[One can also visualize $\hat{f}$ by noting that the subgraph $\{(x, t): \hat{f}(x) \geq t\}$ is the closure of the subgraph of $f$.) We now associate with each bounded function $f$ on $E$ a sequence $\left(f_{\xi}\right)$ of functions as follows:

$$
f_{1}=\hat{f}, \quad f_{2}=\widehat{\hat{\hat{f}-f}+f},
$$

and more generally, if $f_{\xi}$ is defined

$$
f_{\xi+1}=\widehat{\overline{f_{\xi}-f}+f}
$$

and for limit $\lambda, f_{\lambda}$ is defined iff for all $\xi<\lambda f_{\xi}$ is defined and $\sup _{\xi<\lambda} f_{\xi}$ is bounded, and then we set

$$
f_{\lambda}=\widehat{\sup _{\xi<\lambda} f_{\xi}} .
$$

Proposition 2. Let $f$ be a bounded function on $E$. The following are equivalent:

(i) $f \in D B S C$,

(ii) for all $\xi<\omega_{1}, f_{\xi}$ is defined,

(iii) there exists a $\xi<\omega_{1}$ such that $f_{\xi}$ is defined and $f_{\xi+1}=f_{\xi}$ and in this case, if $\xi$ is least so that (iii) holds, the pair $\left(f_{\xi}, f_{\xi}-f\right)$ is the least pair of usc functions $(u, v)$ with $u \geq f$ and $f=u-v$.

Proof. (i) $\Rightarrow$ (ii) As $f$ is in DBSC, on can find $u, v$ in usc with $f=u-v$ and $u \geq f$ (by adding a constant to any solution-this is where the boundedness of the usc functions is used), so that $v \geq 0$. We now prove by induction on $\xi<\omega_{1}$ that $f_{\xi}$ is defined, and $u \geq f_{\xi}$. 
This is true for $\xi=1: f \leq u$ and $u$ is usc so $\hat{f} \leq u$. It is trivial for limit $\lambda$ : if $f_{\xi} \leq u$ for $\xi<\lambda$, then $\sup _{\xi<\lambda} f_{\xi} \leq u$ is bounded, hence $f_{\lambda}=\widehat{\sup _{\xi<\lambda} f_{\xi}}$ is defined and $\leq u$. And for $\xi+1$

$$
f_{\xi} \leq u \Rightarrow f_{\xi}-f \leq u-f=v .
$$

As $v$ is usc, $\widehat{f_{\xi}-f} \leq v$, hence $\widehat{f_{\xi}-f}+f \leq v+f=u$ and finally $f_{\xi+1} \leq u$.

(iii) $\Rightarrow$ (i) The sequence $\left(f_{\xi}\right)$ is clearly increasing, so that the closed subgraphs form an increasing family of closed sets in $E \times \mathbb{R}$, which must stabilize at some $\xi<\omega_{1}$.

(ii) $\Rightarrow$ (iii) It remains to show that if $f_{\xi+1}=f_{\xi}$, the function $f_{\xi}-f$ is usc, for then $f$ is in DBSC and moreover the last assertion is a consequence of the fact proved in (i) $\Rightarrow$ (ii) above. But

$$
\begin{aligned}
f_{\xi}-f & =f_{\xi+1}-f=\widehat{\widehat{f_{\xi}-f}+f}-f \\
& \geq \widehat{f_{\xi}-f}+f-f=\widehat{f_{\xi}-f}
\end{aligned}
$$

and the proof is complete.

Motivated by the preceding proposition, define

$$
r_{D}(f)=\left\{\begin{array}{cc}
\text { least } \xi & \left(f_{\xi} \text { is defined and } f_{\xi+1}=f_{\xi}\right) \\
& \text { if such a } \xi \text { exists } \\
\omega_{1} \quad \text { otherwise }
\end{array}\right.
$$

and

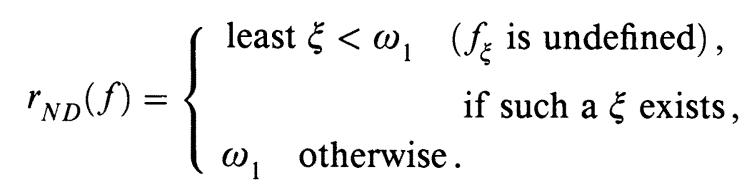

By Proposition $2 r_{D}$ and $r_{N D}$ are ranks on DBSC and its complement, respectively. Note that $r_{D}(f)$ can be, a priori, any ordinal $\geq 1$, whereas $r_{N D}(f)$ is always limit. We will show that $r_{D}$ and $r_{N D}$ are unbounded, on DBSC and $\mathscr{B}_{1}{ }^{1} \backslash$ DBSC, respectively.

Lemma 3. Let $\xi<\omega_{1}$ be given. For each $1 \leq \xi^{\prime}<\omega^{\xi}$ and $\varepsilon>0$, there exists a countable compact set $K$ and a function $f \in \operatorname{DBSC}(K), 0 \leq f \leq 1$, such that (i) $r_{D}(f)=\omega^{\xi}$ and $\left\|f_{\omega^{\xi}}\right\|_{K}=1$, (ii) $\left\|f_{\xi^{\prime}}\right\| \leq \varepsilon$.

Proof. If $\left(K_{n}\right)_{n \in \mathbf{N}}$ and $K_{\infty}$ are countable compact sets, we denote by $S\left(\left(K_{n}\right), K_{\infty}\right)$ the following countable compact set: View $K_{\infty}$ as a subcompact set of $E=\{0,1\}^{\mathbb{N}}$. It is nowhere dense in $E$, hence one easily builds a sequence $\left(U_{n}\right)$ of nonempty pairwise disjoint clopen sets in $E \backslash K_{\infty}$ such that $\overline{\bigcup_{n} U_{n}}=\cup_{n} U_{n} \cup K_{\infty}$. Let $L_{n} \subseteq U_{n}$ be a copy of $K_{n}$, and set $S\left(\left(K_{n}\right), K_{\infty}\right)=K_{\infty} \cup \bigcup_{n} L_{n}$.

We prove Lemma 3 by induction on $\xi$. 
Case 1. $\xi=0$ (so that condition (ii) is void). Let $K=S\left(\left(\left\{x_{n}\right\}\right),\left\{x_{\infty}\right\}\right)$, and

$$
f(x)= \begin{cases}0 & \text { if } x=x_{\infty} \\ 1 & \text { otherwise }\end{cases}
$$

Then $0 \leq f \leq 1, \hat{f}=1$ and $\hat{f}-f$ is usc, so that the conditions are fulfilled.

Case 2. $\xi$ is limit. Let $\xi_{n}$ be strictly increasing with $\xi=\sup _{n} \xi_{n}$, and for each $n$ let $\left(K_{n}, f^{n}\right)$ satisfy (i) and (ii) for $\xi_{n}+1, \xi^{\prime}=\omega^{\xi n}$ and $\varepsilon=1 / n$. Let $K_{\infty}=\left\{x_{\infty}\right\}$, and define $K=S\left(\left(K_{n}\right), K_{\infty}\right)$ and

$$
f= \begin{cases}f^{n} & \text { on (the copy of) } K_{n}, \\ 0 & \text { on } K_{\infty} .\end{cases}
$$

As $K_{n}$ is clopen in $K$, one has for all $\theta$ for which $f_{\theta}$ is defined, $f_{\theta} \uparrow_{K_{n}}=\left(f^{n}\right)_{\theta}$. Now if $\theta \leq \omega^{\xi n},\left\|f_{\theta}^{p}\right\| \leq 1 / p$ for $p \geq n$, hence $\limsup _{y \rightarrow x_{\infty}} f_{\theta}(y)=0$, and as $f\left(x_{\infty}\right)=0$, one easily gets by induction that for $\theta<\omega^{\xi} f_{\theta}$ is defined and $f_{\theta}\left(x_{\infty}\right)=0$. This shows in particular $r_{D}(f) \geq r_{D}\left(f^{n}\right)$ for all $n$, hence $r_{D}(f) \geq \omega^{\xi}$. Now

$$
\sup _{\theta<\omega^{\xi}} f_{\theta}=\left\{\begin{array}{cc}
f_{\omega^{\xi}}^{n} & \text { on } K_{n}, \\
1 & \text { at } x_{\infty},
\end{array}\right.
$$

as $\left\|f_{\omega^{\xi}}^{n}\right\|=1$ on $K_{n}$, so $f_{\omega^{\xi}}$ is defined and $f_{\omega^{\xi}}=\sup _{\theta<\omega^{\xi}} f_{\theta}$. Finally $f_{\omega^{\xi}}-f$ is usc, hence $r_{D}(f)=\omega^{\xi}$ and $\left\|f_{\omega^{\xi}}\right\|=1$, i.e., (i) is satisfied.

To get (ii), let $\xi^{\prime}<\omega^{\xi}$ and $\varepsilon>0$ be given, and choose $n$ big enough so that $\xi^{\prime} \leq \omega^{\xi_{n}}$ and $\varepsilon \geq 1 / n$. Then by the discussion above, the restriction of $f$ to the subcompact $S\left(\left(K_{p}\right)_{p \geq n}, K_{\infty}\right)$, which still satisfies (i), also satisfies $\left\|f_{\omega^{\xi} n}\right\| \leq 1 / n$.

Case 3. The successor case. Let $\xi_{n}$ be strictly increasing with $\sup \xi_{n}=\omega^{\xi}$, and $\left(K_{n}, f^{n}\right)$ satisfy, by the induction hypothesis, (i) and (ii) for $\omega^{\xi}, \xi_{n}$ and $\varepsilon=1 / n$. Define inductively $\left(L_{p}, g^{p}\right)$ by $L_{1}=K_{1}, g^{1}=f^{1}$, and $L_{p+1}=$ $S\left(\left(K_{n}\right), L_{p}\right)$ and

$$
g^{p+1}= \begin{cases}f_{n} & \text { on } K_{n}, \\ g^{p} & \text { on } L_{p} .\end{cases}
$$

Let finally $K=S\left(\left(L_{p}\right),\left\{x_{\infty}\right\}\right)$ and

$$
f=\left\{\begin{array}{cc}
\frac{1}{p} g^{p} & \text { on } L_{p}, \\
0 & \text { at } x_{\infty} .
\end{array}\right.
$$

We now show that $(K, f)$ satisfy (i) for $\xi+1$. For this, we show by induction on $p$ that $(*) r_{D}\left(g^{p}\right)=\omega^{\xi} \cdot p$ and for $k \leq p\left\|g_{\omega^{\xi} \cdot k}^{p}\right\|_{K_{p}}=k$. For $p=1$, $g^{1}=f^{1}$ and $(*)$ is the inductive hypothesis on $f^{1}$. Suppose we know $(*)$ for 
$g^{p}$. As each $K_{n}$ is clopen in $L_{p+1}$, for each $\theta$ for which $g_{\theta}^{p+1}$ is defined, $g_{\theta}^{p+1} \uparrow_{K_{n}}=f_{\theta}^{n}$, and as in case 2 one easily infers, as for $\theta<\omega^{\xi}$

$$
\underset{\substack{y \rightarrow L^{p} \\ y \notin L_{p}}}{\limsup } g_{\theta}^{p+1}(y)=0
$$

if it is defined, that it is defined and satisfies for $\theta<\omega^{\xi}$

$$
g_{\theta}^{p+1}= \begin{cases}f_{\theta}^{n} & \text { on } K_{n}, \\ g_{\theta}^{p} & \text { on } L_{p} .\end{cases}
$$

Now $\left\|g_{\theta}^{p}\right\|_{L_{p}} \leq 1$ for $\theta<\omega^{\xi}$, and $\left\|f_{\omega^{\xi}}^{n}\right\|_{K_{n}}=1$. So

$$
g_{\omega^{\xi}}^{p+1}=\left\{\begin{array}{cl}
f_{\omega^{\xi}}^{n} & \text { on } K_{n}, \\
1 & \text { on } L_{p}
\end{array}\right.
$$

(this is $(*)$ for $k=1$ ),

$$
g_{\omega^{\xi}}^{p+1}-g^{p+1}=\left\{\begin{array}{cl}
f_{\omega^{\xi}}^{n}-f^{n} & \text { on } K_{n}, \\
1-g^{p} & \text { on } L_{p} .
\end{array}\right.
$$

As $0 \leq 1-g^{p} \leq 1$, and $\left\|f_{\omega^{\xi}}^{n}-f^{n}\right\|_{K_{n}} \geq 1-1 / n$, we get

$$
\begin{aligned}
& \widehat{g_{\omega^{\xi}}^{p+1}-g^{p+1}}= \begin{cases}\widehat{f_{\omega^{\xi}}^{n}-f^{n}} & \text { on } K_{n}, \\
1 & \text { on } L_{p},\end{cases} \\
& \widehat{g_{\omega^{\xi}}^{p+1}-g^{p+1}}+g^{p+1}= \begin{cases}\widehat{f_{\omega^{\xi}}^{n}-f^{n}}+f^{n} & \text { on } K_{n}, \\
1+g^{p} & \text { on } L_{p} .\end{cases}
\end{aligned}
$$

And finally, as $1+g^{p} \geq 1 \geq \sup _{n} \sup _{K_{n}} f_{\omega^{\xi}}^{n}$,

$$
g_{\omega^{\xi}+1}^{p+1}= \begin{cases}f_{\omega^{\xi}}^{n} & \text { on } K_{n}, \\ 1+g_{1}^{p} & \text { on } L_{p} .\end{cases}
$$

It then immediately follows by induction that for all $\theta \geq 1, g_{\omega^{\xi}+\theta}^{p+1}$ is defined and

$$
g_{\omega^{\xi}+\theta}^{p+1}= \begin{cases}f_{\omega^{\xi}}^{n} & \text { on } K_{n}, \\ 1+g_{\theta}^{p} & \text { on } L_{p},\end{cases}
$$

so that $(*)$ is verified for $p+1$.

Let us now consider $(K, f)$. Again for any $\theta$ for which $f_{\theta}$ is defined one has $f_{\theta} \uparrow_{L_{p}}=\frac{1}{p} g_{\theta}^{p}$ so that for any $k$, if $f_{\omega^{\xi} \cdot k}$ is defined,

$$
\left\|f_{\omega^{\xi} \cdot k}\right\|_{L_{p}}=\inf \left(1, \frac{k}{p}\right) \text { by }(*)
$$


hence $\lim \sup _{y \rightarrow x_{\infty}} f_{\omega^{\xi} \cdot k}(y)=0$. It easily follows by induction that $f_{\theta}$ is defined for $\theta<\omega^{\xi+1}$, and $f_{\theta}\left(x_{\infty}\right)=0$, and that

$$
f_{\omega^{\xi}+1}=\left\{\begin{array}{l}
\frac{1}{p} g_{\omega^{\xi} \cdot p}^{p} \text { on } K_{p}, \\
1 \text { at } x_{\infty},
\end{array}\right.
$$

is defined, with $f_{\omega^{\xi}+1}-f$ usc, so that (i) is verified.

To get (ii), let $\xi^{\prime}<\omega^{\xi+1}$ and $\varepsilon>0$ be given, and choose $n$ such that $\varepsilon \geq 1 / n$ and $\xi^{\prime}<\omega^{\xi} \cdot n$. Then the restriction $g$ of the preceding function $f$ to $S\left(\left(L_{p}\right)_{p \geq n^{2}},\left\{x_{\infty}\right\}\right)$ still satisfies (i) and moreover

$$
\left\|g_{\omega^{\xi} \cdot n}\right\|=\sup _{p \geq n^{2}}\left\|\frac{1}{n} g_{\omega^{\xi} \cdot n}^{p}\right\|=\sup _{p \geq n^{2}} \frac{n}{p}=\frac{1}{n} \leq \varepsilon \quad \text { by }(*),
$$

and the proof is complete.

Theorem 4. Let $\xi \geq 1$ be a countable ordinal, and $E$ an uncountable compact metrizable space.

(i) There is $f \in \operatorname{DBSC}(E), 0 \leq f \leq 1$, with $r_{D}(f)=\xi$.

(ii) There is $f \in \mathscr{B}_{1}^{1}(E) \backslash \mathrm{DBSC}, 0 \leq f \leq 1$, with $r_{N D}(f)=\omega^{\xi}$

Proof. In both cases, it is enough to find the function $f$ on some countable compact $K$, for one can embed $K$ in $E$, and the function on $E$ obtained by extending $f$ by 0 off $K$ clearly has the same properties.

(i) For $\xi=1, f$ is given by Lemma 3. And if $\lambda$ is limit and (i) holds for $\xi<\omega^{\lambda}$, it also holds at $\omega^{\lambda}$ by using one of the functions of Lemma 3 . So assume (i) is known for $\eta \leq \omega^{\xi}$, and let us prove it for $\omega^{\xi}<\eta \leq \omega^{\xi+1}$. Again the case $\eta=\omega^{\xi+1}$ is given by Lemma 3 . If now $\eta=\omega^{\xi} \cdot n_{0}+\theta$ with $1 \leq n_{0}<\omega$ and $0 \leq \theta<\omega^{\xi}$, the proof is by induction on $n_{0}$.

As in the proof of Case 3 of Lemma 3, let $\left(K_{p}, f^{p}\right)$ satisfy Lemma 3 with $\omega^{\xi}, \xi_{p}$ and $1 / p$, where $\sup _{p} \xi_{p}=\omega^{\xi}$, and for any countable $K_{\infty}$ and $g$ in $\operatorname{DBSC}\left(K_{\infty}\right)$ with $\left\|g_{r_{D}(g)}\right\| \leq 1$, let $K=K\left(K_{\infty}, g\right)=S\left(\left(K_{p}\right), K_{\infty}\right)$ and

$$
f=f\left(K_{\infty}, g\right)=\left\{\begin{array}{ll}
f^{p} & \text { on } K_{p} \\
g & \text { on } K_{\infty}
\end{array} ;\right.
$$

then as in the proof of Case 3 above, one checks by induction that

$$
\text { for } \begin{aligned}
\theta<\omega^{\xi}, \quad f_{\theta} & = \begin{cases}f_{\theta}^{p} & \text { on } K_{p}, \\
g_{\theta} & \text { on } K_{\infty},\end{cases} \\
f_{\omega^{\xi}} & = \begin{cases}f_{\omega^{\xi}}^{p} & \text { on } K_{p}, \\
1 & \text { on } K_{\infty},\end{cases} \\
f_{\omega^{\xi}+\theta} & = \begin{cases}f_{\omega^{\xi}}^{p} & \text { on } K_{p}, \\
1+g_{\theta} & \text { on } K_{\infty} .\end{cases}
\end{aligned}
$$


So for $g=0$ one gets $f \in \operatorname{DBSC}(K), r_{D}(f)=\omega^{\xi}$ and if $g$ is not usc, $f \in \operatorname{DBSC}(K)$ and $r_{D}(f)=\omega^{\xi}+r_{D}(g)$, with $\left\|f_{r_{D}(f)}\right\|=1+\left\|g_{r_{D}}(g)\right\|$. It follows that if $g$ works on $K_{\infty}$ for $\omega^{\xi} \cdot n_{0}+\theta$, for some $n_{0} \geq 0$ and $\theta \leq \omega^{\xi}$, then

$$
f=f\left(K_{\infty}, \frac{1}{\left\|g_{r_{d}(g)}\right\|} \cdot g\right), K\left(K_{\infty}, \frac{1}{\left\|g_{r_{D}(g)}\right\|} \cdot g\right)
$$

work for $\omega^{\xi}+\omega^{\xi} \cdot n_{0}+\theta=\omega^{\xi}\left(n_{0}+1\right)+\theta$ and the proof is complete.

(ii) Again let $\xi_{n}$ be strictly increasing with $\sup _{n} \xi_{n}=\omega^{\xi}$, and consider the pairs $\left(K_{n}, f^{n}\right)$ of Lemma 3 corresponding to $\xi, \xi_{n}$ and $1 / n^{2}$.

Let now $K=S\left(K_{n},\left\{x_{\infty}\right\}\right)$ and

$$
f= \begin{cases}n f^{n} & \text { on } K_{n}, \\ 0 & \text { at } x_{\infty} .\end{cases}
$$

The function $f$ is bounded (between 0 and 1 ), and as $\sup _{K_{n}} f \leq n / n^{2}=1 / n$, $f$ is the uniform limit of the sequence

$$
g^{n}= \begin{cases}f & \text { on } \bigcup_{p \leq n} K_{p}, \\ 0 & \text { elsewhere },\end{cases}
$$

which are in DBSC. So $f \in \mathscr{B}_{1}^{1}$. Now as before $f_{\theta}$ exists for $\theta<\omega^{\xi}$ and $f_{\theta}\left(x_{\infty}\right)=0$, because $\sup _{K_{n}} f_{\xi_{p}} \leq n / n^{2}=1 / n$ for $n \geq p$. But

$$
\sup _{K_{n}}\left(\sup _{p} f_{\xi_{p}}\right)=\sup _{K_{n}}\left(n \cdot \sup _{p} f_{\xi_{p}}^{n}\right)=n
$$

and $\sup _{\theta<\omega^{\xi}} f_{\theta}$ is unbounded, so $f \notin \mathrm{DBSC}$ and $r_{N D}(f)=\omega^{\xi}$.

Remark. We do not know if the $\omega^{\xi}$, s are the only possible values of $r_{N D}$.

\section{TWO FURTHER CHARACTERIZATIONS OF $\mathscr{B}_{1}^{\xi}$}

I. Pseudo-uniform limits. Recall that we defined in $\S 1$, for any sequence $\bar{f}$ of real functions on $E$, an ordinal $\gamma(\bar{f})$, with the properties that $\gamma(\bar{f})<\omega_{1}$ iff the sequence $\bar{f}$ converges quasiuniformly to a function $f$, and $\gamma(\bar{f})=1$ iff the convergence is uniform.

Here we consider the following intermediate notion.

Definition 1. A sequence $\bar{f}$ of real functions on $E$ is pseudouniformly convergent if $\gamma(\bar{f}) \leq \omega$, i.e., for each $\varepsilon>0$, there exists a finite decreasing sequence $K_{0}=E, K_{1}, \ldots, K_{p}, K_{p+1}=\varnothing$ such that any point in $K_{i} \backslash K_{i+1}$ admits a nbhd in $K_{l}$ on which the sequence is $\varepsilon$-uniformly convergent. 
Define now inductively families $\Phi_{\xi}$ of real functions on $E$ by

$$
\Phi_{0}=C(E),
$$

$\Phi_{\xi+1}=\{f: f$ is a pseudouniform limit of a bounded sequence

$$
\text { of functions in } \left.\Phi_{\xi}\right\} \text {, }
$$

and for limit $\lambda$,

$\Phi_{\lambda}=\{f: f$ is a uniform limit of a bounded sequence

$$
\text { of functions in } \left.\bigcup_{\xi<\lambda} \Phi_{\xi}\right\} \text {. }
$$

Theorem 2. (i) $\mathscr{B}_{1}(E)$ is the least family of functions on $E$ containing $C(E)$ and closed under pseudouniform limits of bounded sequences.

(ii) For each $\xi<\omega_{1}, \mathscr{B}_{1}^{\xi}(E)=\Phi_{\xi}$.

Proof. By the definition of the $\Phi$ 's, the closure of $C(E)$ under pseudouniform limits of bounded sequences is $\bigcup_{\xi<\omega_{1}} \Phi_{\xi}$, so (i) is a consequence of (ii).

We prove (ii) by induction on $\xi$. Case $\xi=0$ is trivial. For limit $\lambda$, it is enough to prove that $\mathscr{B}_{1}^{\lambda}$ is the set of uniform limits of functions in $\bigcup_{\xi<\lambda} \mathscr{B}_{1}^{\xi}$. It certainly contains this set. Conversely if $f \in \mathscr{B}_{1}^{\lambda}, f$ can be approximated uniformly by linear combinations of $1_{A}$ 's in $\mathscr{B}_{1}^{\lambda}$. But then (as $\alpha(A)$ is always successor) such $1_{A}$ 's (and their linear combinations) are in $\bigcup_{\xi<\lambda} \mathscr{B}_{1}^{\xi}$ and we are done. So it remains to study the successor case, i.e. to prove that $\mathscr{B}_{1}^{\xi+1}$ is the set of pseudouniform limits of functions in $\mathscr{B}_{1}^{\xi}$.

We first show the following fact: Let $\bar{f}$ be a sequence in $\mathscr{B}_{1}^{\xi}$ with pointwise limit $f$, and $\varepsilon>0$. Then for any closed $P, P_{\varepsilon, f}^{\omega^{\xi}} \subseteq P_{\varepsilon / 3, \bar{f}}^{\prime}$.

To see this, note first that if $V$ is open in $P$, one has for all ordinals $\theta$, $V \cap P_{\varepsilon, f}^{\theta} \subseteq(\bar{V})_{\varepsilon, f}^{\theta}$. So let $x \in P \backslash P_{\varepsilon / 3, \bar{f}}^{\prime}$, and let $V$ be open in $P, x \in V$, be such that for some $n_{0}, \forall m, n \geq n_{0}\left|f_{m}(y)-f_{n}(y)\right| \leq \varepsilon / 3$ for all $y \in \bar{V}$. Then in particular $\left|f_{n_{0}}(y)-f(y)\right| \leq \varepsilon / 3$ for $y \in \bar{V}$. Then by induction, one easily gets that for all $\theta \bar{V}_{\varepsilon, f}^{\theta} \subseteq \bar{V}_{\varepsilon / 3, f_{n_{0}}}^{\theta}$ and, as $f_{n_{0}} \in \mathscr{B}_{1}^{\xi}$,

$$
V \cap P_{\varepsilon, f}^{\omega^{\xi}} \subseteq \bar{V}_{\varepsilon, f}^{\omega^{\xi}} \subseteq \bar{V}_{\varepsilon / 3, f_{n_{0}}}^{\omega^{\xi}}=\varnothing
$$

i.e. $x \notin P_{\varepsilon, f}^{\omega^{\xi}}$, proving the fact.

Suppose now $\bar{f}$ is a bounded sequence in $\mathscr{B}_{1}^{\xi}$, and pseudouniformly converges to $f$. We want to show that $\beta(f) \leq \omega^{\xi+1}$. To see this, let $\varepsilon>0$ be given, and let $K_{0}=E, K_{1}=E_{\varepsilon / 3, \bar{f}}^{i}$, for $i \leq p$, with $E_{\varepsilon / 3, \bar{f}}^{p+1}=\varnothing$. Applying the fact above to each $K_{1}, i \leq p$, we get $\left(K_{i}\right)_{\varepsilon, f}^{\omega^{\xi}} \subseteq K_{i+1}, i \leq p$, and hence $E_{\varepsilon, f}^{\omega^{\xi} \bullet_{l}} \subseteq K_{l}$ for $i \leq p+1$ so that $\beta(f, \varepsilon) \leq \omega^{\xi} \cdot(p+1)$. 
It remains to prove that each $f \in \mathscr{B}_{1}^{\xi+1}$ is in the set $\Phi$ of pseudouniform limits of functions from $\mathscr{B}_{1}^{\xi}$. From Lemma 2.5 , the set $\Phi$ is a vector space.

We first prove the result for characteristic functions. If $1_{A} \in \mathscr{B}_{1}^{\xi+1}$, then $A=$ $\bigcup\left\{F_{\eta}-F_{\eta+1}: \eta\right.$ even, $\left.\eta<\omega^{\xi} \cdot k\right\}$ for some $k \in \mathbb{N}$ and some decreasing sequence $\left(F_{\eta}\right)_{\eta<\omega^{\xi} \cdot k}$ of closed sets. By considering separately the difference corresponding to $\left(F_{\eta}\right)_{\omega^{\xi} \cdot i \leq \eta<\omega^{\xi} \cdot(i+1)}$ for $i<k$, and using that $\Phi$ is closed under sums, it is enough to show $1_{A} \in \Phi$, whenever $A$ is as above with $k=1$. Let then $\xi_{n}$ be strictly increasing with $\sup _{n} \xi_{n}=\omega^{\xi}$, and define $A_{n}=\bigcup\left\{F_{\eta}-F_{\eta+1}: \eta\right.$ even, $\left.\eta<\xi_{n}\right\}$. The functions $1_{A_{n}}$ are in $\mathscr{B}_{1}^{\xi}$, so it is enough to check that $1_{A_{n}}$ pseudouniformly converges to $1_{A}$. It clearly converges to it pointwise. Let $K=\bigcap_{\eta<\omega^{\xi}} F_{\eta}$. We claim that for $\varepsilon<1, E_{\varepsilon,\left(1_{A_{n}}\right)}^{\prime} \subseteq K$, hence, as all functions are 0 on $K, \gamma\left(\varepsilon,\left(1_{A_{n}}\right)\right) \leq 2$. To see this, let $x \notin K$, and $V$ a nbhd of $x$ with $\bar{V} \cap K=\varnothing$. By compactness, $\bar{V} \cap F_{\xi_{n}}=\varnothing$ for some $n$. But then the sequence $\left(1_{A_{n}}\right)_{p \geq n}$ is constant on $\bar{V}$, hence $x \notin E_{\varepsilon,\left(1_{A_{n}}\right)}^{\prime}$.

Let now $f$ be any function in $\mathscr{B}_{1}^{\xi+1}$. Using 2.9 , we can write $f=\sum_{n=0}^{\infty} g_{n}$, with $\left\|g_{n}\right\| \leq 2^{-n}$ for $n \geq 1$, and $g_{n}$ linear combinations of characteristic functions in $\mathscr{B}_{1}^{\xi+1}$, hence in $\Phi$ by the above facts. So let $g_{n}^{p} \rightarrow g_{n}$ pseudouniformly, with $g_{n}^{\dot{p}} \in \mathscr{B}_{1}^{\xi}$, and without loss of generality, $\left\|g_{n}^{p}\right\| \leq 2^{-n}$ for $n \geq 1,\left\|g_{0}^{p}\right\|_{0} \leq\left\|g_{0}\right\|$. Define now $g^{p}=\sum_{n \leq p} g_{n}^{p}$. Then the sequence $\left(g^{p}\right)$ is bounded, and each $g^{p} \in \mathscr{B}_{1}^{\xi}$. Clearly $g^{p} \rightarrow f$ pointwise, so it remains to show that it converges pseudouniformly. Note that for $q, q^{\prime} \geq p>1$, one has

$$
\left|g^{q}-g^{q^{\prime}}\right| \leq\left|\sum_{n \leq p} g_{n}^{q}-\sum_{n \leq p} g_{n}^{q^{\prime}}\right|+4.2^{-p}
$$

and moreover the sequence $\left(\sum_{n \leq p} g_{n}^{q}\right)_{q}$ pseudouniformly converges to $\sum_{n \leq p} g_{n}$. It immediately follows that $\left(g^{q}\right)_{q \in \mathbb{N}}$ also converges pseudouniformly.

II. Alternating series of usc functions. The above characterization was clearly reminiscent of Lebesgue's theorem on analytically representable functions, which inductively analyzes Borel functions using pointwise convergence. The next characterization is reminiscent of the Hausdorff-Kuratowski analysis of $\Delta_{2}^{0}$ sets in terms of differences of closed sets.

First we define the notion of "sum" of an alternating series of usc functions on $E$ : Let $\xi$ be a countable ordinal, and let $\left(f_{\eta}\right)_{\eta<\xi}$ be a sequence of positive usc functions, indexed by $\xi$, and decreasing. The function $\sum_{\eta<\theta}^{*}(-1)^{\eta} f_{\eta}$ is defined, inductively on $\theta \leq \xi$, by

(i) $\sum_{\eta<\theta+1}^{*}(-1)^{\eta} f_{\eta}=\sum_{\eta<\theta}^{*}(-1)^{\eta} f_{\eta}+(-1)^{\theta} f_{\theta}$, where of course

$$
(-1)^{\theta}= \begin{cases}1 & \text { if } \theta \text { is even, } \\ -1 & \text { if } \theta \text { is odd, }\end{cases}
$$


and

(ii) for limit $\lambda \leq \xi$

$$
\sum_{\eta<\lambda}^{*}(-1)^{\eta} f_{\eta}=\sup \left\{\sum_{\eta<\xi}^{*}(-1)^{\eta} f_{\eta}: \xi \text { even }, \xi<\lambda\right\} .
$$

We say that $f$ is the sum of the alternating series $\left(f_{\eta}\right)_{\eta<\xi}$, written $f=$ $\sum_{\eta<\xi}(-1)^{\eta} f_{\eta}$ if for $\xi$ a successor, $f=\sum_{\eta<\xi}^{*}(-1)^{\eta} f_{\eta}$, for $\xi$ a limit, the sequence $\left(f_{\eta}\right)$ decreases to 0 and $f=\sum_{\eta<\xi}^{*}(-1)^{\eta} f_{\eta}$.

[As we may always extend a sequence $\left(f_{\eta}\right)_{\eta<\xi}$ by the constant function 0 as much as we want, without changing the value of $\sum^{*}$, a function $f=$ $\sum_{\eta<\xi}^{*}(-1)^{\eta_{f_{\eta}}}$, for $\xi$ limit and $f_{\eta}$ 's not tending to 0 is still a sum of an alternating series, but of length $\xi+1$. This will be important in exact computations later on.]

Note that the preceding is well defined, for one easily checks by induction that $\sum_{\eta<\theta}^{*}(-1)^{\eta} f_{\eta}$ is bounded by $f_{0}$, so that the taking of suprema in the limit case does not create difficulties. And of course if $f=\sum_{\eta<\xi}^{*}(-1)^{\eta} f_{\eta}$, one has for $\theta$ even, $\theta^{\prime}$ odd $\leq \xi$

$$
0 \leq \sum_{\eta<\theta}^{*}(-1)^{\eta} f_{\eta} \leq f \leq \sum_{\eta<\theta^{\prime}}^{*}(-1)^{\eta} f_{\eta} \leq f_{0} .
$$

Theorem 3. A function $f$ on $E$ is a bounded Baire class 1 function iff $f$ is the sum of a constant and an alternating series of positive usc functions on $E$.

Proof. (i) for direction $\Leftarrow$, it is enough to show that each sum $\sum_{\eta<\theta}^{*}(-1)^{\eta} f_{\eta}$ is Baire class 1 , for $\left(f_{\eta}\right)_{\eta<\theta}$ a decreasing sequence of positive usc functions. This is done by induction on $\theta$. It is clear to $\theta=1$, and obvious at successors. If now $\lambda$ is limit,

$$
f=\sum_{\eta<\lambda}^{*}(-1)^{\eta} f_{\eta}=\sup \left\{\sum_{\eta<\xi}^{*}(-1)^{\eta} f_{\eta}: \xi \text { even }, \xi<\lambda\right\}
$$

is, by the induction hypothesis, a Baire class 2 function, with supergraph $\{(x, t)$ : $f(x) \leq t\}$ in $\boldsymbol{\Pi}_{2}^{0}$. But one immediately checks that

$$
\sum_{\eta<\lambda}^{*}(-1)^{\eta} f_{\eta}=\inf \left\{\sum_{\eta<\xi}^{*}(-1)^{\eta} f_{\eta}: \xi \text { odd }, \xi<\lambda\right\}-\inf _{\xi<\lambda}\left(f_{\xi}\right)
$$

and as $\inf _{\xi<\lambda} f_{\xi}$ is usc, the subgraph $\{(x, t): f(x) \geq t\}$ is $\boldsymbol{\Pi}_{2}^{0}$ too, hence $f$ is Baire class 1 .

(ii) For direction $\Rightarrow$, let $f$ be a bounded Baire class 1 function, which we may assume is $\geq 0$, by adding a constant to it. We now canonically associate to $f$ a series of usc functions, as follows. Let $g_{0}=f, f_{0}=\widehat{g_{0}}$, and by induction

$$
g_{\xi+1}=f_{\xi}-g_{\xi}=\widehat{g_{\xi}}-g_{\xi}, \quad f_{\xi+1}=\widehat{g_{\xi+1}}
$$


and for limit $\lambda$

$$
g_{\lambda}=\inf _{\substack{\xi \text { even } \\ \xi<\lambda}} g_{\xi} \text { and } f_{\lambda}=\widehat{g_{\lambda}}
$$

where $\hat{h}$ denotes the usc regularization of a (bounded) function $h$. To show that the definition makes sense for all ordinals $\xi$, it is enough to check that all $g_{\xi}$ 's are bounded, which is clear, as we take infima at limits. One immediately checks also that the sequence $\left(f_{\xi}\right)$ is decreasing, hence for some countable ordinal $\xi_{0}$ one has $f_{\xi_{0}}=f_{\xi_{0}+1}$. We now show that one must have $f_{\xi_{0}}$ (and hence $\left.g_{\xi_{0}}\right)=0$.

We first prove, by induction, that $g_{\xi}$ is a Baire class 1 function for all $\xi$. This is the hypothesis on $f$ for $\xi=0$, and is obvious at successors. Now if $\lambda$ is limit

$$
g_{\lambda}=\inf _{\substack{\xi \text { even } \\ \xi<\lambda}} g_{\xi}=\inf _{\substack{\xi \text { even } \\ \xi<\lambda}}\left(f_{\xi}-g_{\xi+1}\right)
$$

But note that for any $\xi \widehat{g_{\xi}}-g_{\xi} \leq \widehat{g_{\xi}}$ as $g_{\xi} \geq 0$, hence $\widehat{\widehat{g_{\xi}}-g_{\xi}} \leq \widehat{g_{\xi}}$ and

$$
g_{\xi+2}=g_{\xi}-\left(\widehat{g_{\xi}}-\widehat{\widehat{g_{\xi}}-g_{\xi}}\right) \leq g_{\xi}
$$

so that we get

$$
g_{\lambda}=\left(\inf _{\xi<\lambda} f_{\xi}\right)=\left(\inf _{\substack{\xi \text { odd } \\ \xi<\lambda}} g_{\xi}\right) .
$$

This immediately implies, as in the proof of direction $\Leftarrow$, that $g_{\lambda}$ is Baire class 1.

So In order to prove that $f_{\xi_{0}}$ is 0 , it is enough to show that if $g \geq 0$ is a Baire class 1 function on $E$, and $\hat{g}=\hat{g}-g$, then $g \equiv 0$.

Suppose this is not true, and let $\varepsilon>0$ be such that $\{x: g(x)>\varepsilon\} \neq \varnothing$. Let $K=\overline{\{x: g(x)>\varepsilon\}}$. As $K$ is a nonempty closed set in $E$, there exists an open $V$ in $E$ with $V \cap K \neq \varnothing$ and $\operatorname{osc}(g, V \cap K) \leq \varepsilon$. Let $h=\hat{g}-g$. We claim that $h \leq \varepsilon$ on $V$. If $x \in V$ and $\hat{g}(x) \leq \varepsilon$, then certainly $h(x) \leq \varepsilon$ as $g \geq 0$, so $h \leq \hat{g}$. If now $\hat{g}(x)>\varepsilon$, one must have $x \in V \cap K$, and $\hat{g}(x)=\widehat{\left.g\right|_{K}}(x)$, as $g$ is $\leq \varepsilon$ off $K$. But $\operatorname{osc}\left(\left.g\right|_{K}\right)(x) \leq \varepsilon$ by the choice of $V$, hence $h(x)=\widehat{\left.g\right|_{K}}(x)-g(x) \leq \varepsilon$ too.

So $h \leq \varepsilon$ on $V$; and as $V$ is open in $E, \hat{h} \leq \varepsilon$ on $V$. Now by hypothesis $\hat{h}=\hat{g}$, hence $\hat{g} \leq \varepsilon$ on $V$, contradicting the fact that $V \cap\{g>\varepsilon\} \neq \varnothing$. This shows $g=0$.

So $f_{\xi_{0}}$ (and $\left.g_{\xi_{0}}\right)=0$. It remains to show that $f=\sum_{\eta<\xi_{0}}^{*}(-1)^{\eta} f_{\eta}$. To see this, it is enough to prove by induction that for all $\xi, f=\sum_{\eta<\xi}^{*}(-1)^{\eta} f_{\eta}+$ $(-1)^{\xi} g_{\xi}$

It is clear for $\xi=0\left(f=g_{0}\right)$, and as $g_{\xi+1}=f_{\xi}-g_{\xi}$, it is immediate at successors. 
Finally if for all even $\xi<\lambda$ limit

$$
f=\sum_{\eta<\xi}^{*}(-1)^{\eta} f_{\eta}+g_{\xi}
$$

then

$$
f=\sup _{\substack{\xi<\lambda \\ \xi \text { even }}} \sum_{\eta<\xi}^{*}(-1)^{\eta} f_{\eta}+\inf _{\substack{\xi<\lambda \\ \xi \text { even }}} g_{\xi}=\sum_{\eta<\lambda}^{*}(-1)^{\eta} f_{\eta}+g_{\lambda}
$$

and we are done.

Theorem 3 can be refined to give a characterization of $\mathscr{B}_{1}^{\xi}, \xi<\omega_{1}$ :

Theorem 4. Let $\xi \geq 1$ be a countable ordinal. For $f \in \mathscr{B}_{1}, f$ is in $\mathscr{B}_{1}^{\xi}$ iff $f$ is the sum of a constant and an alternating series of positive usc functions of length $\leq \omega^{\xi}$.

Proof. There is something strange in the proof. If $f \in \mathscr{B}_{1}^{\xi}, f \geq 0$, one may think that the canonical series associated above is such that $\xi_{0} \leq \omega^{\xi}$ (and $\inf _{\eta<\xi_{0}} f_{\eta}=0$ if $\xi_{0}=\omega^{\xi}$ ), but we do not know if this is true. Also, one may think that if $\varepsilon>0$ is given and the $g_{\eta}$ 's are as above, then $g_{\eta} \leq \varepsilon$ off $E_{\varepsilon, f}^{\eta}$. This is immediate for $\eta=1$, and can be proved for $\eta=2$ (by analyzing the proof above that $\left.f_{\xi_{0}}=0\right)$. However, it is false in general for $\eta>2$. So our proof will be quite different.

(i) Proof of direction $\Leftarrow$. We want to show that for all $\xi \geq 1$, if $\left(f_{\eta}\right)_{\eta<\omega^{\xi}}$ is a sequence of usc functions with $f_{\eta} \geq 0, f_{\eta}$ decreasing and $\inf _{\eta} f_{\eta}=0$, then $f=\sum_{\eta}(-1)^{\eta} f_{\eta}$ is in $\mathscr{B}_{1}^{\xi}$. This is done by induction on $\xi$. First we argue that it is enough to prove, assuming the result is known for $\xi$, the following:

Claim. If $\left(f_{\eta}\right)_{\eta<\omega^{\xi}}$ is a decreasing sequence of usc functions with $f_{\eta} \geq 0$, then $f=\sum_{\eta}^{*}(-1)^{\eta} f_{\eta}$ is in $\mathscr{B}_{1}^{\xi+1}$.

Assume this claim has been proved. Suppose $f=\sum_{\eta<\omega^{\xi+1}}(-1)^{\eta} f_{\eta}$ with $f_{\eta} \geq 0$, decreasing, and $\inf _{\eta<\omega^{\xi+1}} f_{\eta}=0$. Define for each $n \in \omega, \eta<\omega^{\xi} f_{\eta}^{n}=$ $f_{\omega^{\xi} \cdot n+\eta}$, and $f^{n}=\sum_{\eta<\omega^{\xi}}^{*}(-1)^{\eta} f_{\eta}^{n}$. Then $f=\sum_{n} f^{n}$, and $\left|f-\sum_{n=0}^{N} f^{n}\right| \leq$ $f_{\omega^{\xi} \cdot(N+1)}$ goes to 0 uniformly (as the $f_{\eta}$ 's are usc). By the claim, each $f^{n}$ is in $\mathscr{B}_{1}^{\xi+1}$, and hence so is $f$, as desired. This gives the successor case. If now $\lambda$ is limit, and the result is known for all $\xi<\lambda$, let $\xi_{n}$ be strictly increasing with $\lambda=\sup \xi_{n}$, and define $f^{n}=\sum_{\eta<\omega^{\xi_{n}}}^{*}(-1)^{\eta} f_{\eta}$ : Again as $\left|f-f^{n}\right| \leq f_{\omega^{\xi} \cdot n}, f^{n}$ converges uniformly to $f$, and by the claim $f^{n} \in \mathscr{B}_{\xi_{n}+1}$, hence $f \in \mathscr{B}_{\lambda}$ and we are done.

So it remains to prove the claim. The proof is based on the following easy fact: Suppose $f, g$ are functions on $E, \varepsilon, \varepsilon^{\prime}>0$ are given and $V$ is an open set in $E$ on which $|f-g| \leq \varepsilon^{\prime}$. Then for any $\eta<\omega_{1}$,

$$
E_{\varepsilon+2 \varepsilon^{\prime}, f}^{\eta} \cap V \subseteq E_{\varepsilon, g}^{\eta} \cap V
$$


This is immediate to prove by induction on $\eta$, using the inequality, for $x$, $x^{\prime} \in V$

$$
\left|f(x)-f\left(x^{\prime}\right)\right| \leq\left|g(x)-g\left(x^{\prime}\right)\right|+2 \varepsilon^{\prime} .
$$

In particular, one gets

$$
E_{\varepsilon+2 \varepsilon^{\prime}, f}^{\beta(\varepsilon, g)} \subseteq\left\{x \in E \text { : there is no nbhd } V \text { of } x \text { with }\|f-g\|_{V} \leq \varepsilon^{\prime}\right\} .
$$

Using this fact, we prove the claim as follows. We assume direction $\Leftarrow$ has been proved for $\xi$, and let $\left(f_{\eta}\right)_{\eta<\omega^{*}}$ be a decreasing sequence of positive usc functions with $f=\sum_{\eta<\omega^{\xi}}^{*}(-1)^{\eta} f_{\eta}$. We want to prove that $f \in \mathscr{B}_{1}^{\xi+1}$. Consider $g=\left(\inf _{\eta<\omega^{*}} f_{\eta}\right)$. This is a usc function, and if $\left(\eta_{k}\right)_{k \in \omega}$ is some strictly increasing sequence of even ordinals with $\sup \eta_{k}=\omega^{\xi}, g$ is the optimal limit of the sequence $\left(f_{\eta_{k}}\right)$, as this sequence is decreasing, by the remarks following Theorem 2.3. So if $\varepsilon>0$ is given, we can find $p \in \mathbb{N}$ and a sequence $K_{0}=E \supseteq K_{1} \supseteq \cdots \supseteq K_{p} \supseteq K_{p+1}=\varnothing$ of compact sets such that for every point $x \in K_{i} / K_{i+1}$, there is a nbhd $V$ of $x$ in $K_{i}$ and some $k$ with $\left\|f_{\eta_{k}}-g\right\|_{V} \leq \varepsilon / 3$. Now notice that for any even $\theta<\omega^{\xi}$, one has

$$
f-\sum_{\eta<\theta}^{*}(-1)^{\eta} f_{\eta} \leq f_{\theta}-g
$$

and moreover, by the induction hypothesis, all the functions $\sum_{\eta<\theta}^{*}(-1)^{\eta} f_{\eta}$ are in $\mathscr{B}_{\xi}{ }^{1}$, so that $\beta\left(\varepsilon / 3, \sum_{\eta<\eta_{k}}^{*}(-1)^{\eta} f_{\eta}\right) \leq \omega^{\xi}$ for all $k$. Applying then the fact above $p+1$ times to all $\left.f\right|_{K_{i}}$, and the corresponding paris of nbhds $V$ and functions $\sum_{\eta<\eta_{k}}^{*}(-1)^{\eta} f_{\eta}$, one immediately gets for all $i \leq p+1 \quad E_{\varepsilon, f}^{\omega^{\xi} \cdot i} \subseteq K_{i}$ hence in particular $E_{\varepsilon, f}^{\omega^{\xi} \cdot(p+1)}=\varnothing$, i.e. $\beta(\varepsilon, f) \leq \omega^{\xi} \cdot(p+1)$. This gives finally $\beta(f) \leq \omega^{\xi+1}$, as desired.

(ii) Proof of $\Rightarrow$. Suppose first $f=1_{A} \in \mathscr{B}_{1}^{\xi}$. Then $A=\bigcup\left\{F_{\theta}-F_{\theta+1}: \theta\right.$ even, $\theta<\eta_{0}$ for some $\eta_{0}<\omega^{\xi}$ and a decreasing sequence $\left(F_{\theta}\right)_{\theta<\eta_{0}}$ of closed sets. The functions $\left(1_{F_{\theta}}\right)_{\theta<\eta_{0}}$ are usc, and one immediately checks that $1_{A}=$ $\sum_{\eta<\eta_{0}}^{*}(-1)^{\eta} 1_{F_{\eta}}$. (The definition was chosen to get this automatically.)

Suppose now $f \in \mathscr{B}_{1}^{\xi}$, and assume, without loss of generality, that $f \geq$ 0 . By 2.9 , one can find for each $N>2$ a sequence $1_{A_{1}}, \ldots, 1_{A_{N-2}}$ in $\mathscr{B}_{1}^{\xi}$ such that $g=(\|f\| / N) \sum_{k=1}^{N-2} 1_{A_{k}}$ satisfies $0 \leq g \leq f \leq g+\frac{2}{N}\|f\|$. Note that if $\eta_{N}$ even less than $\omega^{\xi}$ is such that $\alpha\left(1_{A_{k}}\right) \leq \eta_{N}$, and by the above result $1_{A_{k}}=\sum_{\eta<\eta_{N}}^{*}(-1)^{\eta} 1_{F_{\eta}^{k}}$, then $g_{\eta}=(\|f\| / N) \sum_{k=1}^{N-2} 1_{F_{\eta}^{k}}$ is usc, the $g_{\eta}$ 's are decreasing and $g=\sum_{\eta<\eta_{N}}(-1)^{\eta} g_{\eta}$. Moreover $0 \leq g_{0} \leq\|f\|(1-2 / N)$. Applying this result to $f$ and $N=4$, one gets $f_{1}$ and $\left(f_{\eta}^{1}\right)_{\eta<\eta_{1}}, \eta_{1}<\omega^{\xi}$, with $0 \leq f_{1} \leq f \leq f_{1}+\|f\| / 2$ and $f_{1}=\sum_{\eta<\eta_{1}}^{*}(-1)^{\eta} f_{\eta}^{1}$ with $0 \leq f_{0}^{1} \leq\|f\| / 2$. Applying it to $f-f_{1}$ and $N=4$, one gets $f_{2}$ and $\left(f_{\eta}^{2}\right)_{\eta<\eta_{2}}, \eta_{2}<\omega^{\xi}$, with 
$0 \leq f_{1}+f_{2} \leq f \leq f_{1}+f_{2}+\|f\| / 4$ and $f_{2}=\sum_{\eta<\eta_{2}}^{*}(-1)^{\eta} f_{\eta}^{2}$ with $0 \leq f_{0}^{2} \leq$ $\|f\| / 4$ and continuing this way, one gets $f_{k}$ and $\left(f_{\eta}^{k}\right)_{\eta<\eta_{k}}, \eta_{k}<\omega^{\xi}$, with $0 \leq f_{1}+f_{2}+\cdots+f_{k} \leq f \leq f_{1}+f_{2}+\cdots+f_{k}+\|f\| / 2^{k}$ and $f_{k}=\sum_{\eta<\eta_{k}}^{*}(-1)^{\eta} f_{\eta}^{k}$ with $0 \leq f_{0}^{k} \leq\|f\| / 2^{k}$. Let $\theta=\sum_{k} \eta_{k} \leq \omega^{\xi}, \theta_{i}=\sum_{k<l} \eta_{k}$, and for $\theta_{i} \leq \eta<\theta_{i+1}$ let $f_{\eta}=f_{\eta-\theta_{i}}^{i}+\sum_{j>i} f_{0}^{J}$. This sum is well defined, for the series is uniformly converging, and $f_{\eta}$ is thus usc. Moreover $\left(f_{\eta}\right)_{\eta<\theta}$ is decreasing. And finally as

$$
\sum_{\theta_{t} \leq \eta<\theta_{i+1}}^{*}(-1)^{\eta} f_{\eta}=\sum_{\eta<\eta_{t}}^{*}(-1)^{\eta} f_{\eta}^{i}=f_{i},
$$

one gets $f=\sum_{\eta<\theta}^{*}(-1)^{\eta} f_{\eta}$. And as $\sum_{i>k} f_{0}^{i} \leq 2^{-k+1}\|f\|$ goes to 0 with $k$, $f=\sum_{\eta<\theta}(-1)^{\eta} f_{\eta}$ and we are done.

\section{OPTIMAL CONVERGENCE AND DERIVATIVES}

In many cases, Baire class 1 functions occur in analysis through natural approximation processes by continuous functions. The rank of convergence $\gamma$ introduced in $\S 1$ thus provides a quantitative measure on how the natural approximation process is the "best possible" one.

For example if $f$ is usc, then $f$ is the infimum of a decreasing sequence of continuous functions, and as we have seen in $\S 2$ (after Theorem 2.3), for bounded usc functions this is indeed optimal. Similarly if $f$ is of bounded variation on the circle $\mathbb{T}, \mathrm{T}$. Ramsamujh has verified that the Fourier series of $f$ has convergence rank $\leq 2$, so that if one assumes $f(x)=(f(x-)+f(x+)) / 2$ at each discontinuity point $x$ of $f$, the convergence of the series is optimal.

We study here the more involved case of derivatives on $[0,1]$. Note that by a result of Petruska and Laczkovich [P-L], any Baire class 1 function on the Cantor set can be extended to a derivative on $E$, so that the Baire class 1 ranks are unbounded on the set $\mathscr{D}$ of derivatives on $[0,1]$.

Of course if $f$ is a derivative and $F$ is a primitive of $f$, a very natural approximation of $f$ is given, for a given sequence $\bar{h}=\left(h_{n}\right) \rightarrow 0$, by the sequence

$$
F^{h_{n}}(x)=\frac{F\left(x+h_{n}\right)-F(x)}{h_{n}} .
$$

We will see below that these approximations may be very far from giving the best approximation, i.e. optimally converge to the derivative $f$.

However, one has a positive result in this direction.

Theorem 1. Let $f$ be a bounded derivative on $[0,1]$, and $F$ its primitive. There exists a sequence of positive step functions $h_{n}(x)$ on $[0,1]$, uniformly converging to 0 , such that the differences

$$
F_{n}^{\bar{h}}(x)=\frac{F\left(x+h_{n}(x)\right)-F(x)}{h_{n}(x)}
$$

optimally converge to $f$. 
[Here we always view $f$ and $F$ as defined on all of $\mathbb{R}$, with $f=0$ outside $[0,1]$, when the formulas involve points not in $[0,1]$.

Proof. It is a simple consequence of Theorem 2.3. Starting with $h_{n}=1 / n$, we get a sequence $\varphi_{n}$ of functions optimally converging to $f$, with

$$
\varphi_{n}(x)=\sum_{k=1}^{k_{n}} \lambda_{k}^{(n)} m_{k}^{(n)}\left(F\left(x+\frac{1}{m_{k}^{(n)}}\right)-F(x)\right)
$$

for some $0 \leq \lambda_{k}^{(n)} \leq 1, \sum \lambda_{k}^{(n)}=1$, and $n \leq m_{1}^{(n)}<\cdots<m_{k_{n}}^{(n)}$. And we may assume $m_{k_{n}}^{(n)}<m_{1}^{(n+1)}$. For each $x$ on $[0,1]$,

$$
\psi_{x}(h)=\frac{F(x+h)-F(x)}{h}
$$

is continuous between $a^{(n)}=1 / m_{k_{n}}^{(n)}$ and $b^{(n)}=1 / m_{1}^{(n)}$, hence has range some interval $\left[a_{x}^{(n)}, b_{x}^{(n)}\right]$. The values $\psi_{x}\left(1 / m_{i}^{(n)}\right)$ are in this interval for all $i \leq k_{n}$, hence so must be $\varphi_{n}(x)$. So for some $h$ in $\left[a^{(n)}, b^{(n)}\right]$

$$
\varphi_{n}(x)=\psi_{x}(h)=\frac{F(x+h)-F(x)}{h} .
$$

By continuity, each $x \in[0,1]$ is in a nbhd $U_{x}$ such that for some $h \in$ $\left[a^{(n)}, b^{(n)}\right]$ and $y \in U_{x}$,

$$
\left|\frac{F(y+h)-F(y)}{h}-\varphi_{n}(y)\right|<\frac{1}{n},
$$

and by compactness we can find $0=x_{0}^{(n)}<x_{1}^{(n)}<\cdots<x_{p_{n}}^{(n)}=1$ and $h_{1}^{(n)}, \cdots, h_{p_{n}}^{(n)}$ in $\left[a^{(n)}, b^{(n)}\right]$ such that for $y \in\left[x_{i}^{(n)}, x_{i+1}^{(n)}\right]$

$$
\left|\frac{F\left(y+h_{i}^{(n)}\right)-F(y)}{h_{i}^{(n)}}-\varphi_{n}(y)\right|<\frac{1}{n} .
$$

The function $h_{n}(x)$ defined by the $h_{1}^{(n)}, \ldots, h_{p_{n}}^{(n)}$ clearly converges uniformly to 0 in $n$, and moreover as $\left\|F_{n}^{\bar{h}}-\varphi_{n}\right\| \leq 1 / n, \gamma\left(\left(F_{n}^{\bar{h}}\right)\right)=\gamma\left(\left(\varphi_{n}\right)\right)$ and $F_{n}^{\bar{h}}$ converges optimally to $f$.

We do not know if the functions $h_{n}(x)$ can be chosen continuous in $x$. The next result shows that constants do not work in general.

Theorem 2. Let $\xi$ be a countable ordinal. There exists a bounded derivative $f$ on $[0,1]$ with $\beta(f)=2$ (hence $f \in \mathscr{B}_{1}^{1}$ ) such that if $F$ is a primitive of $f$ and $\left(h_{n}\right)_{n \in \mathbb{N}}$ is any sequence in $(0,1)$ converging to 0 , the sequence

$$
F^{h_{n}}(x)=\frac{F\left(x+h_{n}\right)-F(x)}{h_{n}}
$$

of differences satisfies $\gamma\left(\left(F^{h_{n}}\right)\right)>\xi$. 
Proof. The functions $F$ (and $f$ ) are constructed by induction on $\xi$. We will prove in fact the following: For any interval $[a, b]$ in $[0,1]$ and any $\xi$, there exists a differentiable $F=F_{[a, b]}^{\xi}$ such that

(i) $F \geq 0, F=0$ off $[a ; b],\|F\| \leq b-a$,

(ii) $F$ is differentiable and $\left\|F^{\prime}\right\|<C$ (some fixed constant),

(iii) $F^{\prime}$ is continuous off some closed subset $A$ of $[a, b]$, and is identically 0 on $A$,

(iv) for all $h_{n}>0, h_{n} \rightarrow 0, b \in[a, b]_{1 / 2,\left(F^{h_{n}}\right)}^{\xi}$.

Note that (iii) ensures that $\beta\left(F^{\prime}\right) \leq 2$, and (iv) ensures $\gamma\left(\left(F^{h_{n}}\right)\right) \geq \xi$.

Case 1. $\xi=1$. By scaling, we let $a=0, b=1$. Let $x_{1}=1 / 2, x_{m}=1-1 / 2^{m}$, so that $x_{m+1}-x_{m}=1 / 2^{m+1}$. Let

$$
\begin{aligned}
& y_{m}=x_{m}+\frac{2^{-(m+2)}}{m+2}, \quad z_{m}=x_{m}+\frac{2^{-(m+1)}}{m+1}, \\
& t_{m}=\frac{2^{-(m+1)}}{m+1}, \quad v_{m}=x_{m}+\frac{2^{-(m+2)}}{m+2}+\frac{2^{-(m+1)}}{m+1}
\end{aligned}
$$

and choose on $\left[x_{m}, x_{m+1}\right] \quad F$ to be continuously differentiable, 0 at $x_{m}$ and on $\left[v_{m}, x_{m+1}\right]$ with derivative 0 at $x_{m}$, and equal to $t_{m}$ on $\left[y_{m}, z_{m}\right]$, with $F^{\prime}$ bounded by some constant $C\left(=3\right.$ e.g., as $\left.t_{m} /\left(y_{m}-x_{m}\right)<3\right)$. Finally, let $F=0$ outside the intervals $\left[x_{m}, x_{m+1}\right]$. Clearly $F$ is differentiable off 1 . But if $1 / 2<x<1$, then for some $m, x_{m} \leq x<x_{m+1}$ and

$$
\left|\frac{F(x)-F(1)}{x-1}\right| \leq \frac{t_{m}}{2^{-(m+1)}}=\frac{1}{m+1}
$$

goes to 0 as $m \rightarrow \infty$, and $F$ is differentiable at 1, with $F^{\prime}(1)=0$. So (i), (ii) and (iii) (with $A=\{1\}$ ) are satisfied. To see (iv), let $h_{n} \rightarrow 0, h_{n}>0$, and let $V$ be a nbhd of 1 and $p \in \mathbb{N}$. Choose $n_{0}$ large enough so that $x_{m} \in V$ for $m \geq n_{0}$, and $n_{0} \geq p$. As $h_{n} \rightarrow 0$, let $n_{1}$ be such that $h_{n_{1}} \leq 2^{-\left(n_{0}+1\right)} /\left(n_{0}+1\right)$, and let $m \geq n_{0}$ be such that

$$
\frac{2^{-(m+2)}}{m+2}<h_{n_{1}} \leq \frac{2^{-(m+1)}}{m+1} .
$$

The point $x_{m}$ is in $V$, and $F^{h_{n_{1}}}\left(x_{m}\right)=t_{m} / h_{n_{1}} \geq 1$, whereas (as $F^{\prime}\left(x_{m}\right)=0$ ) one can find $p>m$ with $F^{h_{p}}\left(x_{m}\right) \leq 1 / 2$, so that $1 \in[0,1]_{1 / 2,\left(f^{\left.h_{n}\right)}\right.}^{\prime}$. This proves Case 1.

Case 2. Let use prove now the successor case (the limit case being similar, although a bit simpler). So let $\xi$ be given, and assume $F_{[a, b]}^{\xi}$ have been constructed, with properties (i)-(iv) for $\xi$. Again by scaling we work on $[0,1]$. 
Let $F^{1}$ be the function defined above (for $\xi=1$ ), and let $w_{m}=x_{m+1}-10^{-m}$ (so that $\left.w_{m} \geq v_{m}\right)$. Add to $F^{1}$, for each $m$, the function $F_{\left[w_{m}, x_{m+1}\right]}^{\xi}=F_{m}^{\xi}$

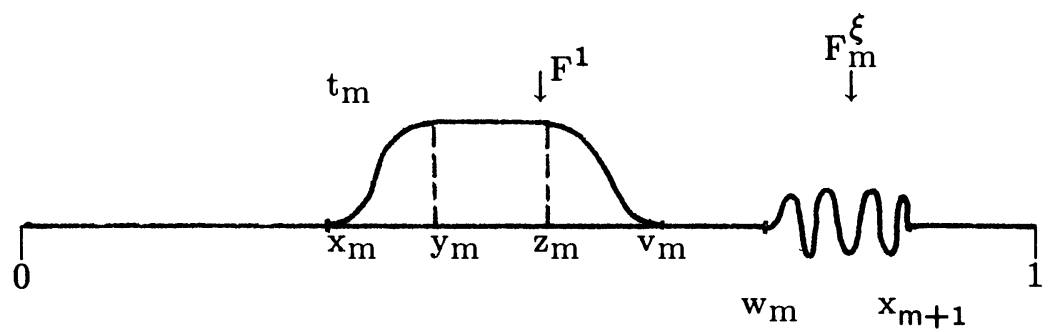

This gives $F=F^{\xi+1}$. We now check that it works. (i) is immediate, and (ii) too, expect at 1 . But as $\left\|F_{m}^{\xi}\right\| \leq 10^{-m}$, one sees as before that $F$ is differentiable at 1 with $F^{\prime}=0$. (iii) Let $A_{m} \subseteq\left[w_{m}, x_{m+1}\right]$ be the closed set on which $\left(F_{m}^{\xi}\right)^{\prime}$ is 0 and off which it is continuous. Then $A=\bigcup_{m} A_{m} \cup\{1\}$ is closed, $\left(F^{\xi+1}\right)^{\prime}$ is continuous off it, and 0 on it, so (iii) is checked. For (iv), let again $h_{n}>0, h_{n} \rightarrow 0$ be given. By the inductive hypothesis, we know that $x_{m+1} \in\left[w_{m}, x_{m+l}\right]_{1 / 2,\left(\left(F_{m}^{\xi}\right)^{h_{n}}\right)}^{\xi}$ so that one also gets $x_{m+1} \in[0,1]_{1 / 2,\left(F^{h_{n}}\right)}^{\xi}$, and as this last set is closed, 1 is in it too. But then the proof we gave before for $F_{1}$-which used only the $x_{m}$ 's-works as well to show that $1 \in[0,1]_{1 / 2,\left(F^{h_{n}}\right)}^{\xi+1}$.

Remarks. 1. A slight change in the previous constructions easily gives, for each $\xi$, an $F$ with the same properties and satisfying also, for any $h_{n} \rightarrow 0, k_{n} \rightarrow 0$, $h_{n}, k_{n}>0$, if

$$
F^{h_{n}, k_{n}}=\frac{F\left(x+h_{n}\right)-F\left(x-k_{n}\right)}{h_{n}+k_{n}}, \quad \gamma\left(\left(F^{h_{n}, k_{n}}\right)\right) \geq \xi .
$$

[Replace the basis $F^{1}$ on $\left[x_{m}, x_{m+1}\right]$ by a function like:

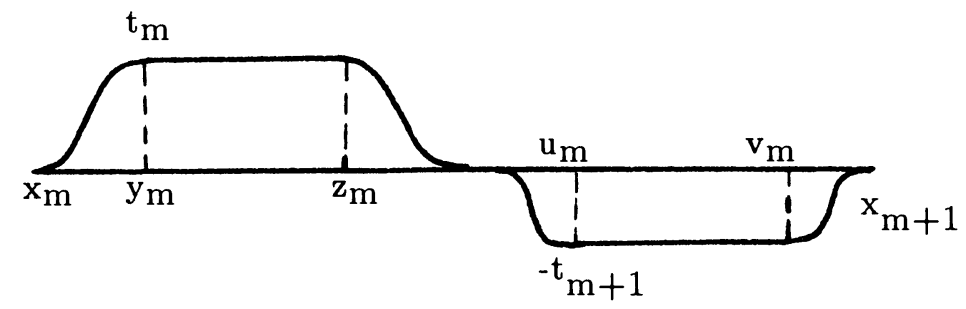

and argue similarly].

2. For each differentiable function $F$, one can define a differentiability rank $|F|_{D}$ as in Kechris and Woodin [K-W]. It is easy to see that for each $\left(F_{n}^{\bar{h}}(x)\right)$, with $h_{n}(x)$ step functions uniformly converging to 0 , one has $\gamma\left(\left(F_{n}^{\bar{h}}(x)\right)\right) \leq$ $|F|_{D}$. We do not know if $|F|_{D}$ is the supremum of these $\gamma\left(\left(F_{n}^{\bar{h}}(x)\right)\right)$. 


\section{REFERENCES}

[B] J. Bourgain, On convergent sequences of continuous functions, Bull. Soc. Math. Belg. Sér. B 32 (1980), 235-249.

[C] G. Choquet, Remarques à propos de la démonstration d'unicité de P.-A. Meyer, (appendice), Séminaire de Théorie du Potentiel, $6^{e}$ année, exposé no. 8, Inst. Hautes Etudes Sci. Paris, 1961-62.

[G-H] D. C. Gillespie and W. A. Hurwicz, On sequences of continuous functions having continuous limits, Trans. Amer. Math. Soc. 32 (1930), 527-543.

[H-O-R] R. Haydon, E. Odell and H. P. Rosenthal, Certain subclasses of Baire-1 functions with Banach space applications, circulated notes.

[K-W] A. S. Kechris and W. H. Woodin, Ranks of differentiable functions, Mathematika 33 (1986), 252-278.

[O-R] E. Odell and H. P. Rosenthal, A double-dual characterization of separable Banach spaces containing $l^{1}$, Israel J. Math. 20 (1975), 3-4.

[P-L] G. Petruska and M. Laszkovich, Baire 1 functions, approximately continuous functions and derivatives, Acta Math. Hungar. 25 (1974), 189-212.

[Z] Z. Zalcwasser, Sur une propriété du champ des fonctions continues, Studia Math. 2 (1930), 63-67.

Department of Mathematics 253-37, California Institute of Technology, Pasadena, CALIFORNia 91125

Equipe d'Analyse, Université Paris Vi, 4 Place Jussieu, 75252-Paris Cedex 05, France 